\title{
LES ÉTUDES HYDROLOGIQUES AU CAMEROUN
}

\author{
P. DARNAULT \\ Directeur des Travaux Publics ef des Chemins de Fer du Cameroun \\ Ingénieur-Docteur
}

\section{AVANT-PROPOS}

Si la géographie physique et la météorologic du Cameroun sont, dans l'ensemble, assez bien connues, il n'en est pas de mème du régime de ses cours d'eau. Quelques études préliminaires, mais très superficielles, ont bien été abordées et notamment certains accidents spectacilaires comme les chutes d'Eréa sur la Sanaga, la chute du N'jock sur le Nyowg, la chute d'Ekom sur Ie N'kam, de la Vina près de N'gaoundere ei de la Lobe près de Kribi avaient déjà été signalés.

De nombreux administrateurs en ont fait mention sur leurs relevés d'itinéraires et dans leurs 'rapports; la carte Moisel qui, pour l'époque à laquelle elle a été faite, représentait un travail extrêmement important, mentionne un certain nombre de rapides et de chutes ainsi que l'altitude de divers points. Plus récemment, un administrateur, M. Licrorx, a dressé une liste des sources natronées de la région BanyoN'gaoundere (1) et l'Ingénieur des Mines de VRILs a étudié en 1935 certains cours d'eau do la région du Wouri (2). Par ailleurs, le personnel du Service des Mines sous la direction de l'Ingénieur en Chef Havgov a établi une étude extrêmement intéressante (3) relative à l'assèchement éventuel du Lac Tchad par la capture du Logone au profit de la Benoue. Enfin, M. SLor, Ingénieurr, et divers agents de notre service ont amorcé en 1944 une première étude des chutes de la Sanga à Edea.

Cependant, du point de vue des ressources hydrologiques et hydroélectriques, le territoire du Cameroun est particulièrement intéressan! puisque, en dehors d'accidents isolés, les p'rin. cipaux fleuves descendent brusquement de plusieurs centaines de mètres avant de se jeter dans la mer, présentant ainsi une succession de chutes alors qu'ils présentent un débit considérable dú,

(1) Liste des Docodje utilisés dans la région do N'gaoundere par LAcrorx, Bulletin n" 2 de la Société des Etades Camerounaises.

(2) Rapport de la mission de recherches pétrolifères IE VPIES 1935

(3) Etude de lassechement possible du lac Thad pal P. Havgou, Ing. on chef des Mines, 1945 . d'une part, aux précipitations atmosphériques très abondantes, et d'autre pari, à la grande suliface de leur bassin versant.

Nous nous proposons, dans cette première étude, de définir les principaux besoins en eau que nécessitera le développement économique du Cameroun dans les prochaines années; d'indiquer. les caractéristiques des divers bassins ainsi que l'état actuel de nos connaissances; enfin compte tenu des besoins par ordre d'urgence, nous chercherons quelle organisation simple permettrait de doter le territoire de la documentation précise qui lui fait complètement défaut.

\section{I. - LES BESOINS EN EAU}

Nous nous proposons de passer successivement en revue les principaux besoins en eau.

\section{DISTRIBUTION D'EAU POTABLE}

Distinguons tout d'abord l'eau d'alimentation des populations des villes principales et des villes secondaires.

Les deux grandes villes de Douala et de Yaounde ont actuellement des distributions d'eau très insuffisantes; on s'est contenté, en effet, jusqu'à maintenant d'établii: des distributions d'eau pour les populations européennes, en négligeant les besoins des populations africaines. Il est bien certain que l'ćvolution rapide de ces territoires commande d'adopte'r d'autres méthodes et de tabler sur l'ensemble de la population à desservir, tant européenne qu'africaine, pour les futures distributions d'eau.

Pour la ville de Douala qui compte actuellement 1.000 Européens et 40.000 Africains, nous estimons que les besoins en eau, y compris les besoins du port, doivent ètre calculés sur la base de 15.000 mètres cubes d'eau purifiée par jour, compte tenu des extensions futures; pour Yaounde dont la population actuelle est de 700 Européens et 18.000 Africains, il faudra comp. ter sur 10.000 mètres cubes d'eau par jour, compte tenu des besoins des industries locales. 
et des exteinsions probables dans les prochaines années.

A Douala, on dispose d'une couche aquifère située entre 10 et 15 mètres de la surface du sol, couche que l'on retrouve, à plusieurs kilomètres autour de la ville, régulièrement alimentée par les pluies dont le régime très régulier est extrèmement important (4 mètres d'eau par an). Uni nouveau sondage exécuté au Nord-Est de la ville a recoupé cette nappe entre 9 et 16 mètres. La dist ibution actuelle donne 2.500 mètres-cubes par jour, par deux usines alimentées par une série de forages avec pompes à émulsion. On satisfera aux besoins futurs de la ville, soit par d'autres usines du mème type alimentées par de nouveaux forages, soit par la captation à une dizaine de kilomètres de la ville d'un cours d'eau dont le débit d'étiage correspondrait à 175 litres par seconde.

Pour Yaounde la question est plus complexe ; la ville est actuellement dépourvue d'eau, puisqu'il n'est guère distribué que 250 mètres cubes par jour. Kous avons fait procéder au jaugeage de tous les cours d'eau de la région en période d'ćliage, et il faudra vraisemblablement envisager une prise d'eau à plusieurs kilomètres de la ville sur un cours d'eau important. Il ne semble pas, en efret, que l'on puisse espérer trouver le débit nécessaire à l'aide de forages dans le voisinage immediat de la ville où le terrain particulièrement accidenté recoit des précipitations beaucoup plus faibles qu’à Douala, soit environ 2 mètres par an.

Le Territoire vient de passer un contrat avee la Société «Eaux et Assainissement» pour l'exécution des recherches et l'élaboration des projets complets de distribution d'eau de ces deux villes, élude comprenant en outre les stations de purification et des réseaux complets de distribution.

En ce qui concerne les villes secondaires : N'kongsamba, Fourbam, Kribi, Ebolowa, M'balmayo, Batouri, N'gaoundere, Dschang, des p'rojets complets devont être également étudiés par la suite si l'on veut éviter des dangers de contamination par des eaux souillées. Dans toutes ces régions, d'ailleurs, l'eau ne manque pas; nous envisageons de faire entreprendre, dès que possible, cies études à ce sujet.

Pour les localités importantes de Garoua et Maroua, cette dernière comptant 20.000 habitants africains, la question de l'eau se pose d'une façon différente et il faudra certainement entreprendre des campagnes de forages pour donner, a la population qui manque d'eau pendant plusieurs mois, un débit suffisant à ses beoins. De telles recherches sont d'autant plus importantes qu'en plus de l'alimentation humaine, la ques- tion se pose de l'alimentation en eau du bétail très nombreux dans les régions situées au nord de la Benoue.

\section{ELEVAGE}

Nous ne dirons qu'un mot des sources natronées que la nature a mises généreusement à la disposition du bétail dans la région de Banyo, Tigniere, N'gaoundere où une station de pompage permet l'alimentation de plusieurs milliers de têtes de bétail à la fois. Les bergers conduisent leurs troupeaux régulièrement à ces sources et après quelques jours les bêtes ayant terminé leur cure retournent à leurs pâturages. C'est un spectacle très curieux que de voir d'immenses troupeaux se ruant littéralement vérs ces sources dès qu'ils en approchent : les premiers jours ils boivent souvent des quantités d'eau trop importantes, mais ils réduisent d'eux-mêmes la dose les jours suivants.

Dans la région de N'gaoudere on compte environ 40 sources salées dont certaines n'ont qu'un très faible débit.

La captation de ces sources, l'analyse de leurs eaux et la construction d'abreuvoirs devront etre entreprises car l'abondance du bétail conslitue la principale richesse des hauts plateaux de l'Adamaoua.

\section{IRRIGATION}

On peut dire que, sur presque toute sa superficie, le Cameroun peut se dispenser des irrigations car les pluies sont partout suffisamment régulières et importantes. Toutefois, on devra. faire cependant une exception pour le triangle situé au Nord de la Benoue et dont Maroua occupe le centre. Là, le développement de l'agriculture exigerait de nombreux forages car les besoins en eau sont considérables pendant la saison sèche. En outre, dans cette même région les soins du betail exigeraient la construction de deeping-tanks qu'il faudra alimenter en eau.

\section{INDUSTRIE}

Si l'on envisage maintenant les besoins industriels, on comprend qu'ils aient été négligés jusqu'ici puisque les seules industries du Cameroun se réduisaient à quelques petites savonneries ou scieries situées dans des régions où les précipitations sont tres fortes. Or, dans les prochaines années on peut espérer voir se développer des industries très importantes exigeant de fortes quantités d'eau soit directement pour le traitement des produits : usines de cellulose par exemple, soit indirectement pour la production 
d'énergie électrique à bon marché d'origino hydraulique.

La région de Douala et toute la zone cotiere appelées à un développement économique plus intense deviendront rapidement des consommateurs de courant électrique importants ainsi que les usines nouvelles : scieries, fabriques de contreplaqués et panneaux défibrés, etc.

\section{GENIE CIVIL}

Enfin, l'écuipement du territoire va imposer la construction de nombreux ouvrages de génie civil qui ne peurent être conçus rationnellement que si l'on connaît d'une facon précise le régime des cours d'eau qu'ils doivent f'ranchir, ce qui n'a pas été le cas jusqu'ici, il faut bien le dire. On voit encore très souvent des ouvrages de débouché insuffisant et des ponts submergés ou emportés aux hautes eaux, ce qui est dû principalement a une méconnaissance complète de l'hydrologie assez particulière des régions tropicales.

\section{NAVIGATION}

Nous ne signalons, que pour mémoire, les besoins de la navigation (comnaissance des seuils, propagation des crues) qui intéressent cependant la navigation sur les biefs de la Benoue, de la Sanaga, du Nyong, du Wouri et du N'tem.

On voit par ce tableau largement esquissé combien sont variés les besoins en eau du terriloire; aucun développement économique ou social ne sera désormais possible si l'on n'attache pas à cette question l'importance qu'elle mérite et cela implique évidemment des études qui n'avaient pas été faites jusqu'ici. Compte tenu des documents que nous ayons pu rassemble'r et que nous avons signalés en tète de cette étude, ainsi cque des premiers travaux entrepris par nos soins depuis le mois d'août 1945, nous avons cherché à dresser un bilan de nos connaissances présentes.

\section{II. - DESCRIPTION DES PRINCIPAUX BASSINS}

Le Territoire Francais du Cameroun, qui s'étend entre les paralleles 2 et 13 de latitude nord et les meridiens 9"34 et $16 \% 15$ de longitude est, ne correspond pas à une région ou à un ensemble de régions bien déterminé; il est le résultat d'une suite de découpages au hasard des traités, mais du point de vue orographique on peul distinguer les bassins ou fractions de bassins suivants.

\section{FLEUVES PRINCIPAUX}

- Le bassin de la Sanaga, principal fleuve qui traverse le territoire d'est en ouest et qui est un des seuls dont tout le bassin est en territoire du Cameroun.

- le bassin du fleuve Nyong.

- le bassin du fleuve N'tem dont une grande partie est située dans les territoires de l'Afrique Equatoriale Française et de la Guinée Espagnole.

- le bassin du fleuve Wouri dont une très faible partie est englobée dans le territoire du Cameroun Britannique.

\section{BENOUE SUPERIEURE, AFFIUENT DU NIGER}

La source de la Benoue se trouve dans le territoire du Cameroun, mais son bassin supérieur se trouve partagé avec l'Afrique Equatoriale

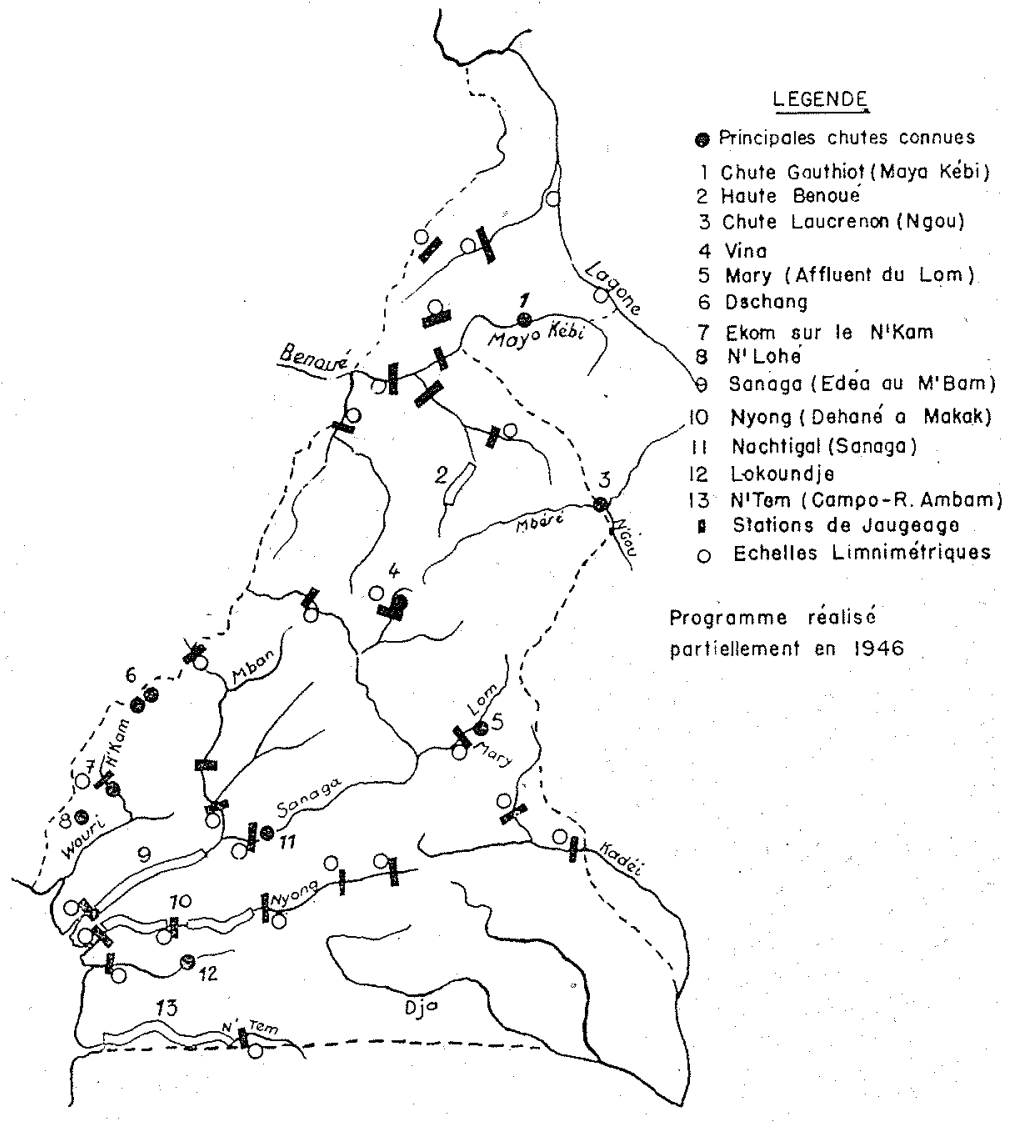

P.D.

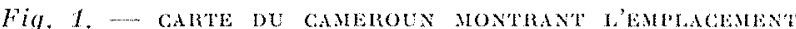

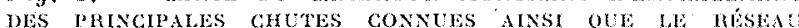

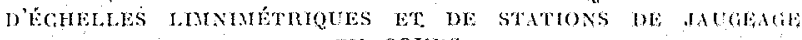
EN covis. 
Française (affluents du Mayo-Kebbi). Le reste ciu bassin jusqu'au confluent avec le Niger, c'esta-dire la plus grande partie forme une partie de la Nigeria Britannique.

Fleuves cotiers secondaires (Lokounji, N'kenke, Lobe).

Tributaires du Logone et du Congo.

Enfin, stur les frontières Nord et Sud du Cameroun, un certain nombre de cours d'eau de l'Afrique Equatoriale Francaise prennent leur solurce :

- Au nord quelques affluents du Logone.

A l'est, le Kadel, afluent de la Sanaga.

- Au sud, la Dja, afluent également de la Sanaga.

La figure 1, montre la répartition de ces divers bassins et indique la situation des principales chutes actuellement connues.

\section{CHAPITRE I}

\section{Bassin de la Sanaga}

Le bassin versant de la Sanaga a une surface de 135.000 kilomètres carrés et l'on peut voir, d'apres la carte au 2.000.000 jointe, extraite de la carte Noisel (fig. 2) que ce fleuve long de \$18 kilomitres draine parfaitement tout son bassin. Il est formé d'abord par le Djerem qui recoit rive droite le Meng. Au confluent du Lom, affluent rive gauche, la Djerem devient la Sanaga qui ne recoit plus qu'un seul affluent très important, le N'bam rive droite, avant de se jeter dans l'Oéan Atlantique.

Gràce à quelques altitudes connues et aux nouvelles observations, nous avons pu dresser un essai de profil en long de la Sanaga et de ses

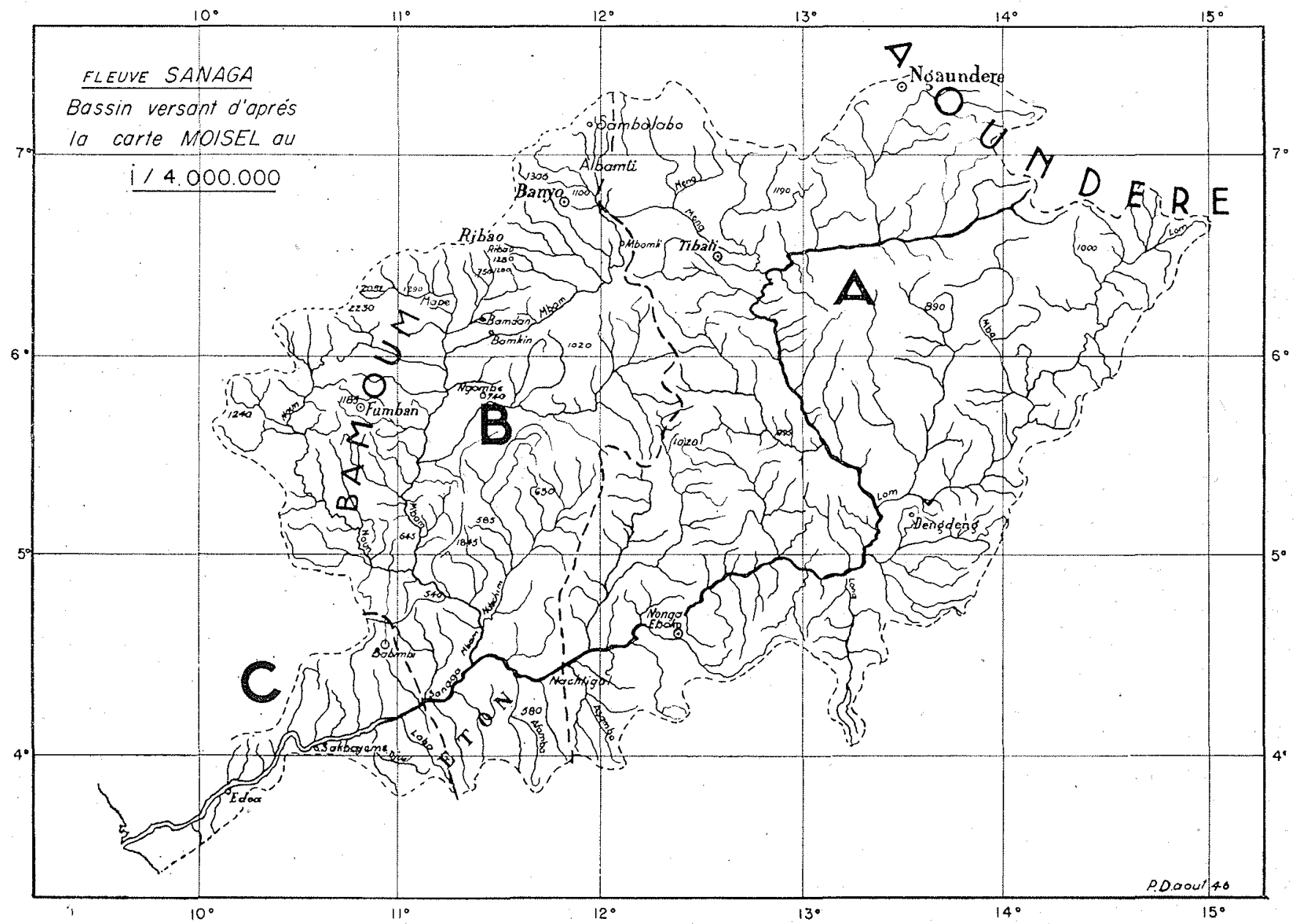

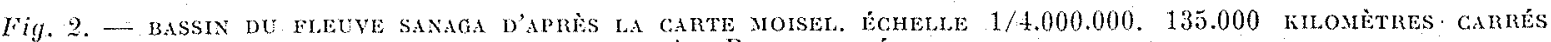

A. BASSIN SUPÉRIEUR

B. BASST MOYEN

C. BASSIN mF́́RIEUtR. 


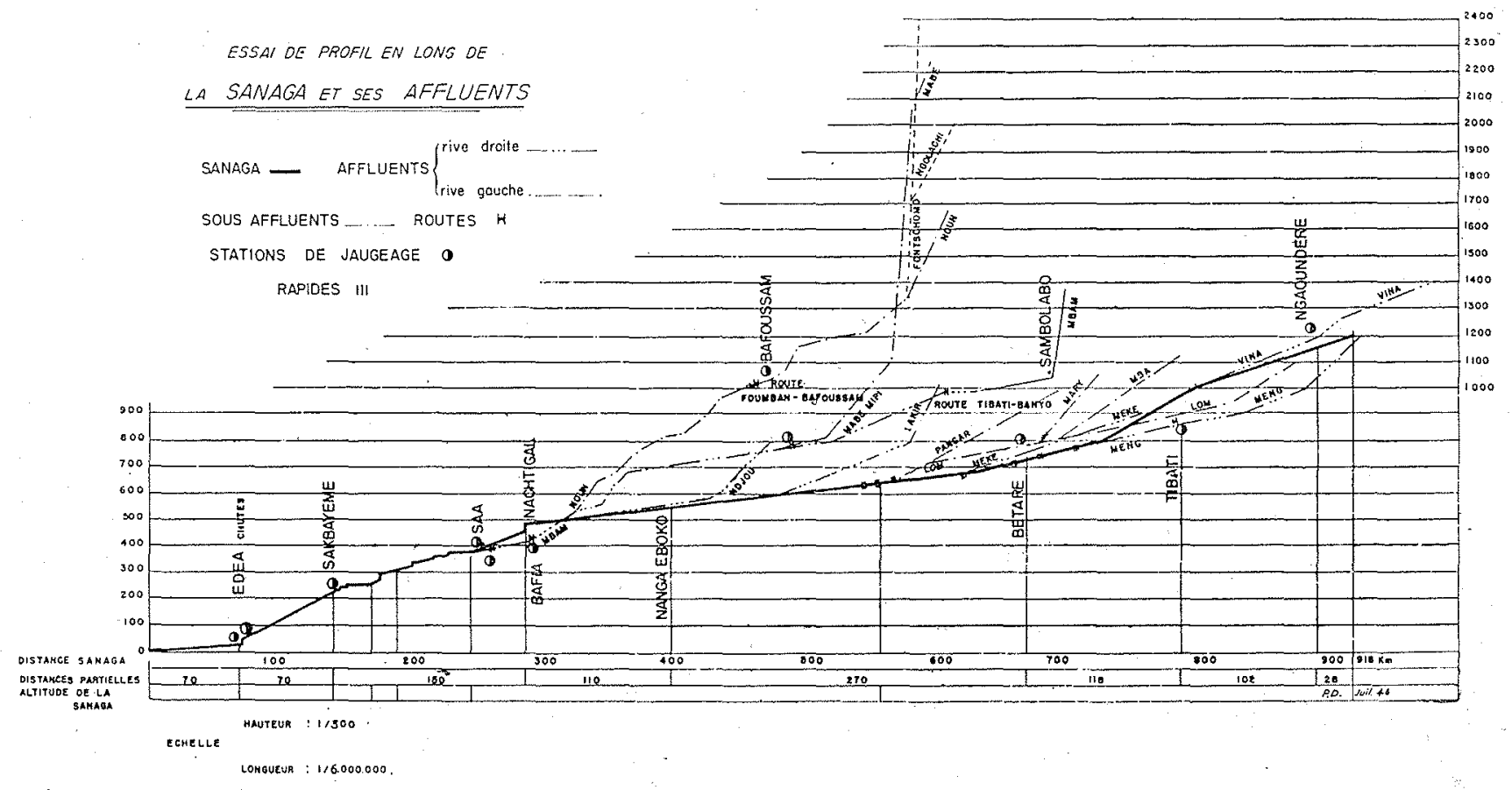

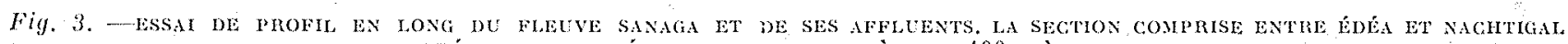
PRÉSENTE UNE DÉNIVLLATION DE PRÈS DE 400 MÈTRES.

aflluents (fig. 3) qui ne représente -- nous insistons sur ce point - qu'une approximation. Jusqu'au confluent du Lom (cote 630 environ) le fleuve descend du plateau central de l'Adamaoua avec une forte pente. Puis jusqu'aux chutes de Nachtigal (cote 480 ) la pente est faible $(0 \mathrm{~m} .40 \mathrm{par} \mathrm{km})$ et le fleuve qui a recu tous seiz aflluents importants, sauf le M'Bam est navigable sur quelques sections de son cours.

Apris le barage naturel d'une dizaine do mètres de hauteur, formé par les chutes Nachtigal, le fleuve recoit rive droite son affluent le plus important, le M'bam, puis il s'engage dans une vallée encaissée et par une succession de rapides et de chutes descend jusqu'à la mer sans que son débit s'accroisse d'apports d'affuents importants.

Ainsi entre le confluent du M'bam et Edea å 70 kilomètres de l'embouchure, soit sur $190 \mathrm{kms}$, la différence de niveau est de 370 mètres et cette section du cours constitue un résérvoir d'énergic hydroélectrique considérable.

Nous ne possédons malheureusement encoro que bien peu de renseignements sur le débit et les éléments caractéristiques; coefficient d'écoulement, débit caractéristique d'étiage par kilomètre carré.

Un certain nombre d'échelles limnimétriques ont été posées (fig. 1) et des jaugeages exécutés. mais les difficultés de toutes sortes propres aux pays tropicaux avec un personnel difficile a former font que les premiers résultats qui nous sont parvemus n'ont guère de valeur scientifique.

Gràce au service météorologique très bien organisé $(t)$ le territoire dispose déjà d'un réseau de stations pluviométriques relativement dense. D'autre part, en aval des chutes d'Edéa, des échelles d'étiage lues régulièrement donnent des indications que nous avons reportées à la planche ci-contre. On voit par l'examen des courbes 1944-1945 et début 1946 que se dessine une cerlaine régularité dans les niveaux.

\section{ETIAGE}

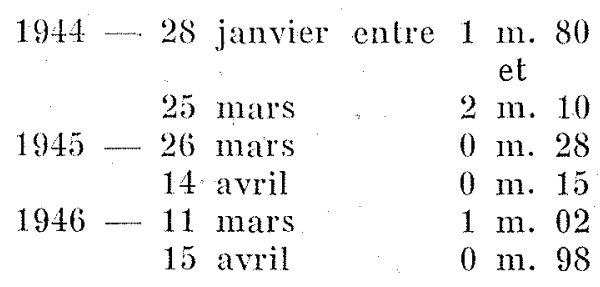

MAXIMUM DE CRUES

$$
\begin{gathered}
1944 \text { - } 27 \text { octobre } \\
1945 \text { - } 10 \text { au } 24 \text { octobre } 70 \\
22 \text { octobre } \quad 7 \text { m. } 70
\end{gathered}
$$

(4) Résultats dobservations : Mar. Preo et Bauory, Insenieurs méteorologicpes. 


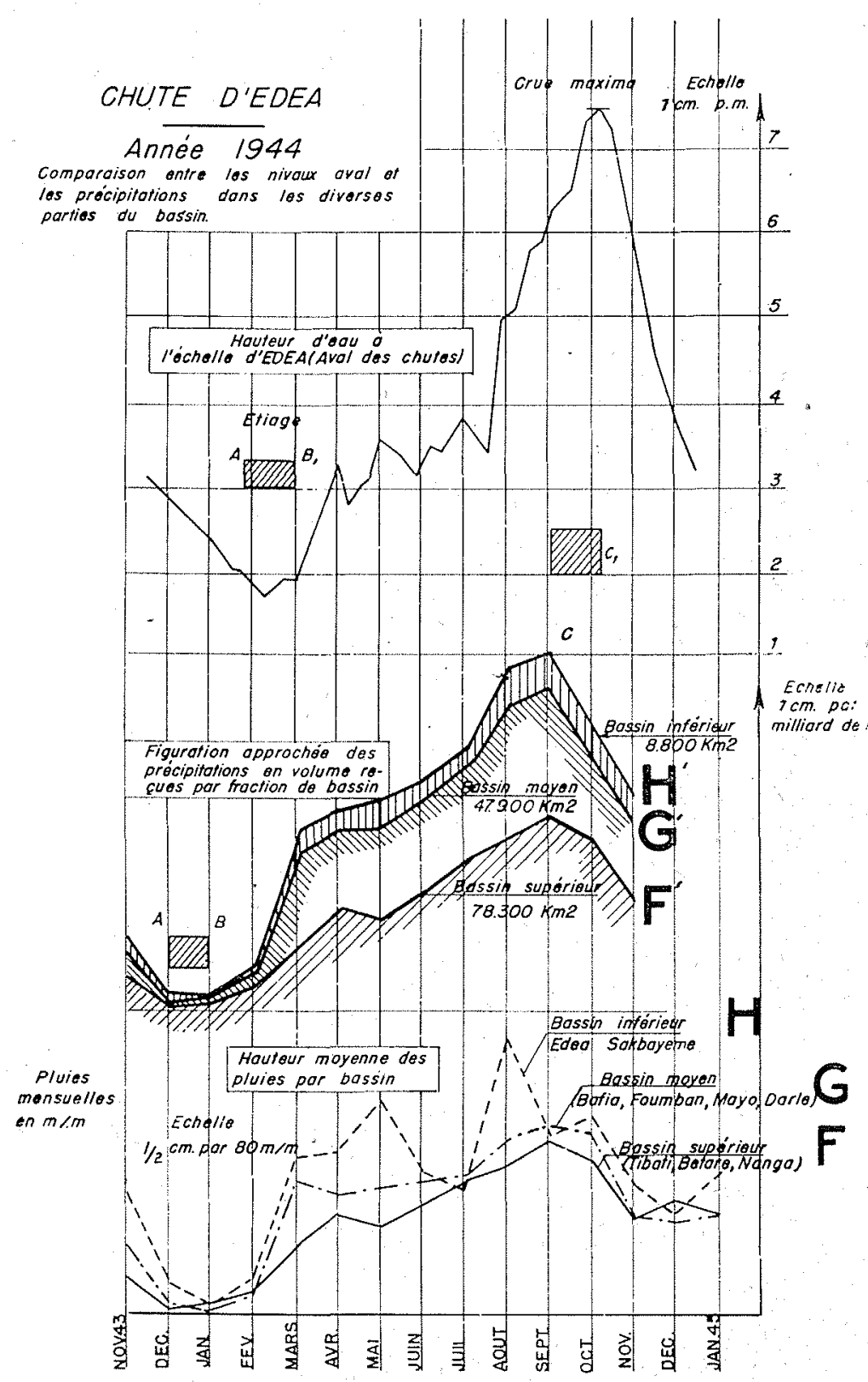

Fig. 4. - COURBes DE CRUES DE la SANAGA pouR L'ANNÉ 1944. COMPARAISON AVEC: LES PRÉCIPITATIONS PIUVYOMETTROUES DANS L.ES DIVERSES PARTIES DC BASSIN

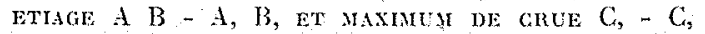

On n'observe qu'une seule crue dans l'année en octobre et elle est due surtout aux précipilations dans les parties supérieure et moyenne du bassin.

Nous avons cherché à faire ressortir d'une manière très approximative pour l'année 1944 le décalage de l'étiage et de la crue maximum par rapport aux précipitations.

Sur le mème graphique (fig. 4) : la courbe $F$ représente lintensité movenne des pluies mensuelles dans la partie supérieure du bassin, soit $78.300 \mathrm{~km}^{2}$,

les ordonnées de la courbe $F^{\prime}$ sont proportionnelles au produit de l'intensité moyenne des pluies par la superficie de la zone intéressée,

de mème les cou'rbes $G G$ ' concernant la partie moyenne B du bassin, soit $47.900 \mathrm{~km}^{2}$ et

les courbes $H H^{\prime}$ la partie inférieure du bassin, soit $8.800 \mathrm{~km}^{2}$.

On voit que l'étiage à Edéa situé à $70 \mathrm{~km}$. de l'embouchure, représenté par la zone A.B. de la courbe supérieure du graphique (Février-Mars) présente un retard de deux mois environ par rapport aux minima de pluies dans les diverses fractions du bassin (zone A B) correspondant au mois de décembre.

Par contre, le maximum de crue à Edéa (C) (27 octobre) est en retard de trente-sept jours par rapport au maximum des précipitations en volume (C) recues par fractions de bassins.

Il est à remarquer que quoique le maximum de pluies dans la partie inférieure du bassin ait lieu en août son influence est négligeable, car la superficie du bassin correspondante à ce régime est très faible.

\section{$D E B I T$}

Le débit mesuré très grossièrement en aval des chutes d'Edéa a été trouvé à l'étiage 1946 de 300 à 350 mètres cubes seconde. Un autre jaugeage exécuté aux plus hautes eaux en septembre 1945 avait permis de chiffrer le débit de crue entre 3.500 et 4.000 mètres cubes seconde, ce qui co'respond à des débits caractéristiques par kilomètre carré de bassin versant d'environ 2,5 litres à l'étiage et 25 à 30 litres aux hautes eaux. D'autres jaugeages peu précis exécutés à Nachtigal et sur le M'bam ont donné des résultats voisins, 2 à 4 litres par kilomètre car'ré de bassin versant à l'étiage.

\section{PUISSANGE HYDROELECTRIQUE}

Revenons à l'examen du profil en long. On voit que certains affluents présentent des zones de trìs grande pente dans lesquelles se trouvent des chutes d'équipement économique, mais ces points sont très disséminés dans le territoire du bassin : rivière Mary dans la région minière de Betare-Cya, hauteur 68 mètres. Par contre, l'attention doit se porter, à notre avis, sur la région de très forte pente qui s'étend du confluent du M'bam aux chutes d'Edéa. Si l'on s'en tient aux débits approximatifs observés, on voit que la puissance thérique dans cette zone varic 


\section{ESSAI DE PROFIL EN LONG}

\section{DU NYONG ET SES AFFLUENTS}

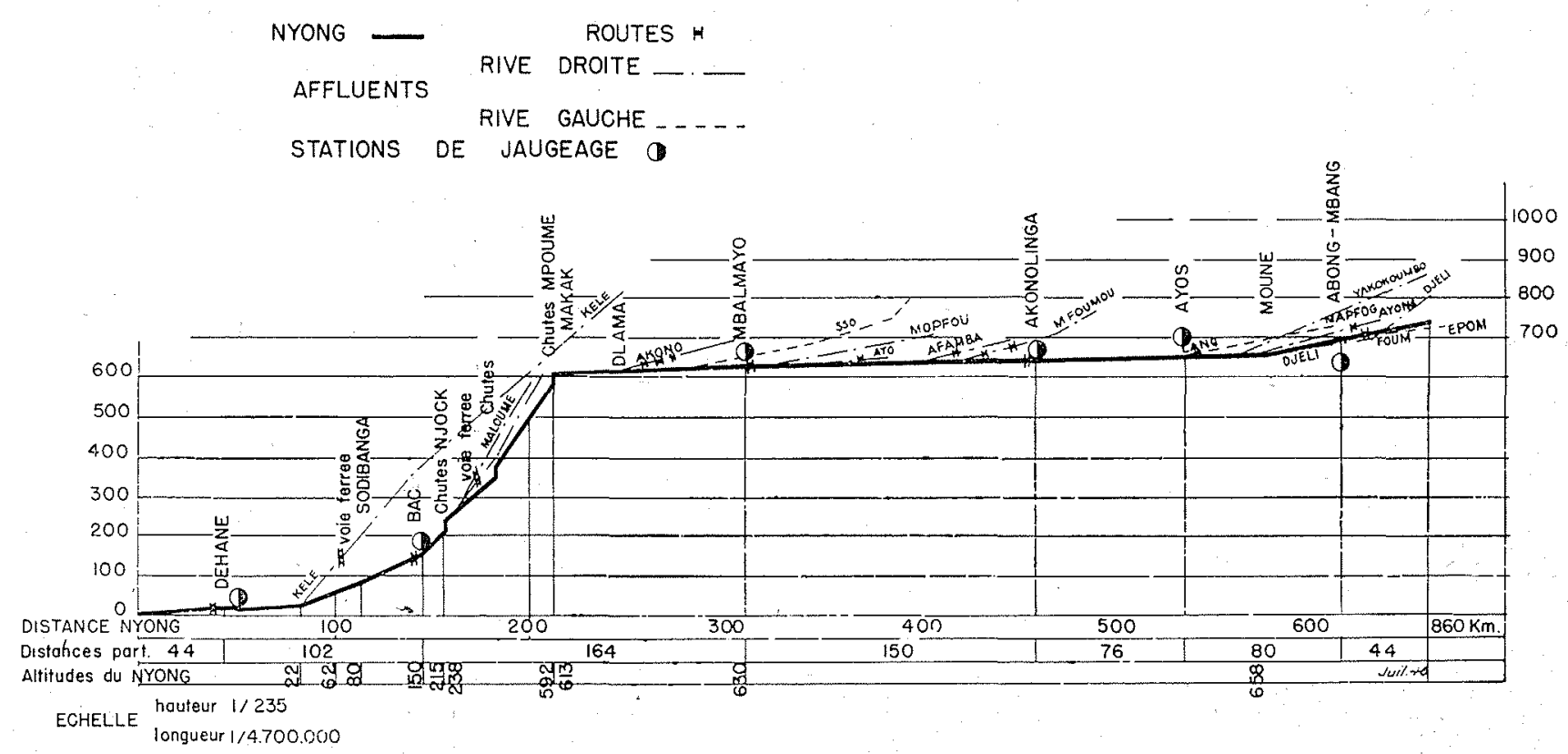

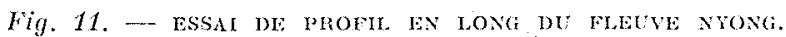

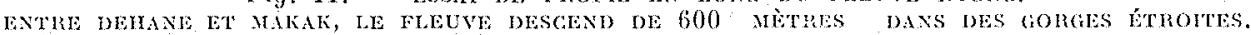

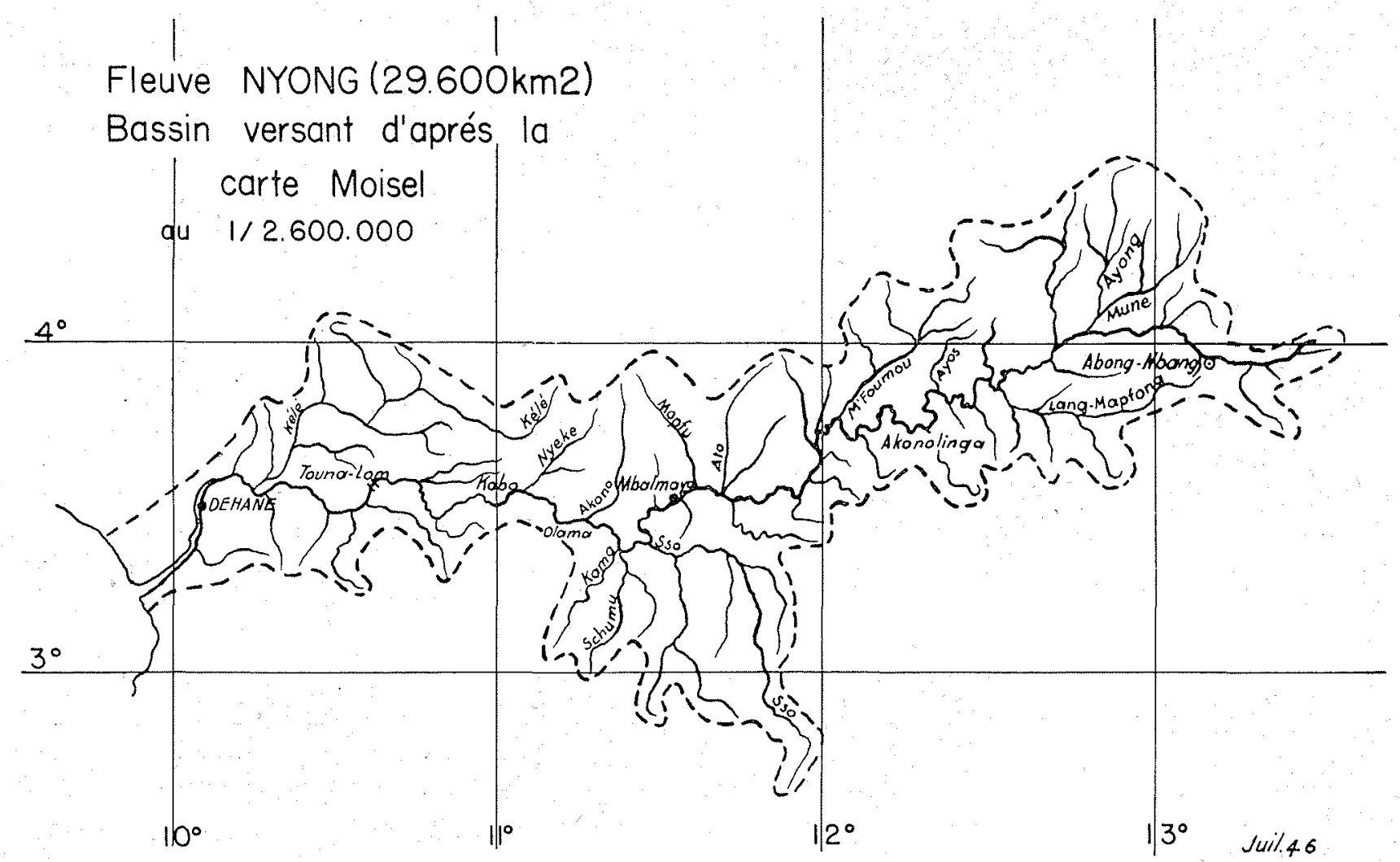

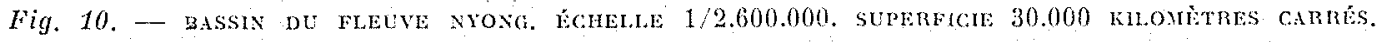




\section{UTILISATION DES CHUTES}

\section{DE LA SANAGA ET D'EDEA}

En vie d'assurer la mise en valeur économique de la région de Douala et de la zone còtière, le Territoire du Cameroun s'était préoccupé dès 1944 d'étudier les chutes du fleuve Sanaga à Edéa.

En aoùt 1945 , le Territoire a passé un contrat pour l'étude d'une usine hydroélectrique alimentée par ces chutes. Après quelques mois d'études sur place, pendant la période des basses eaux, un avant projet a été établi et sera terminé en septembre 1946. Les étapes prévues sont:

$$
\begin{aligned}
& \text {--- } 1^{\mathrm{re}} \text { étape : } 15 \text { à } 20.000^{\mathrm{cv}} \mathrm{c} \text {. } \\
& \text { - 2 étape : } 40.000 \mathrm{cr} \text {. } \\
& \text { - 32 etape : } 60.000 \mathrm{cv} \text {. }
\end{aligned}
$$

La figure 6 montre la disposition d'ensemble de ces chules : le fleuve retenu par une barrière rocheuse forme à l'amont une nappe de plusieurs

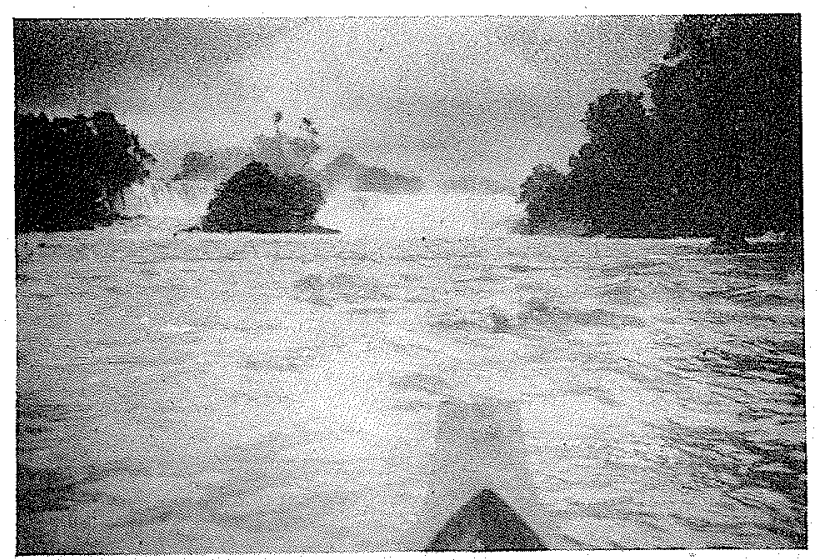

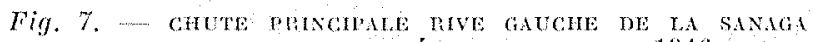

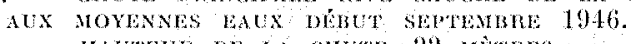
Hatere be a chete, 22 merers

kilomètres de largeur qui s'écoule en cascades par une multitude de bras se rejoignant a l'aval pour n'en former que deux principaux traversés par les deux grands ponts de chemin de fer d'Edéa.

La nature des roches a fait l'objet de premieres études (5), et il semble qu'aucune difficulté ne doive se présenter pour l'établissement des ouvrages de Génie Civil, on se trouve en effet en présence de gneiss à biotite solides et frais, très superficiellement altérés par places.

Des lignes à haute tension transporteront lénergie à Douala et Kribi et assureront la fourniture d'énergie aux divers Services Publics du 'Territoire ainsi qu'aux industries.

(5) Rapporl préliminare do M. Porsas, IngénienrAdjoint des Mines.

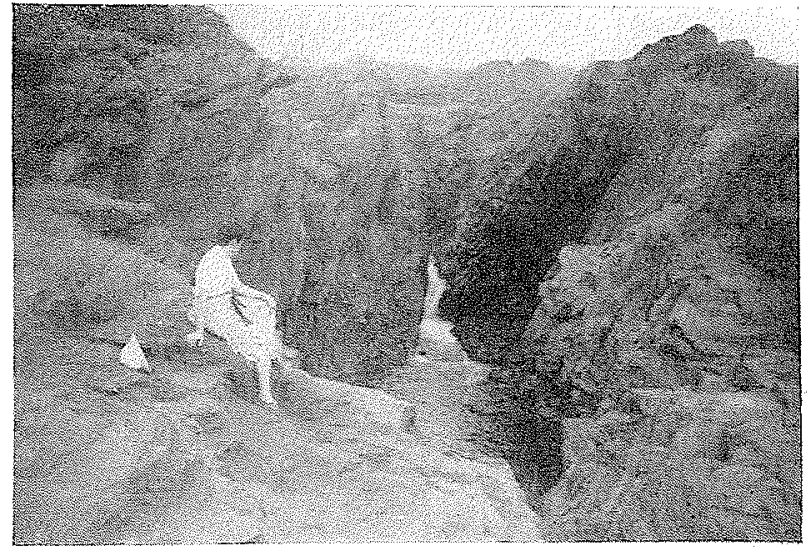

Fig. 8. CHETE DE LA RIVE DROITE DE LA SANAGA A EDEA. A L'ÉTIAGE UN FILET D'EAU COULE AU FOND D'UNE GORGL ETROTT. $H=25$ METRES, AUx FAUTES EAUX LE DËBT A CET ENDROT' ATTELNT $1.500 \cdot \mathrm{M} / \mathrm{SEC}$.

Enfin des études, confiées aux Services Techniques S.N.C.F., ont été entreprises concernant la possibilité d'électrification des réseaux des chemins de fer du Cameroun (ligne Nord et ligne Centre).

II sera ainsi possible dès que les résultats de cette étude seront connus de prendre une décision à ce sujet, compte tenu du développement économique escompté.

Les photographies 7,8 et 9 représentent 'les chutes d'Edéa (rive droite et rive gauche).

\section{CHAPITRE II}

\section{Bassin du Nyong}

Le Nyong a un bassin versant de $30.000 \mathrm{~km}$ ? qui s'étend en longueur parallèlement à la Sanaga sans recevoir d'affluent important (fig. 10). On voit sur l'essai de profil en long,

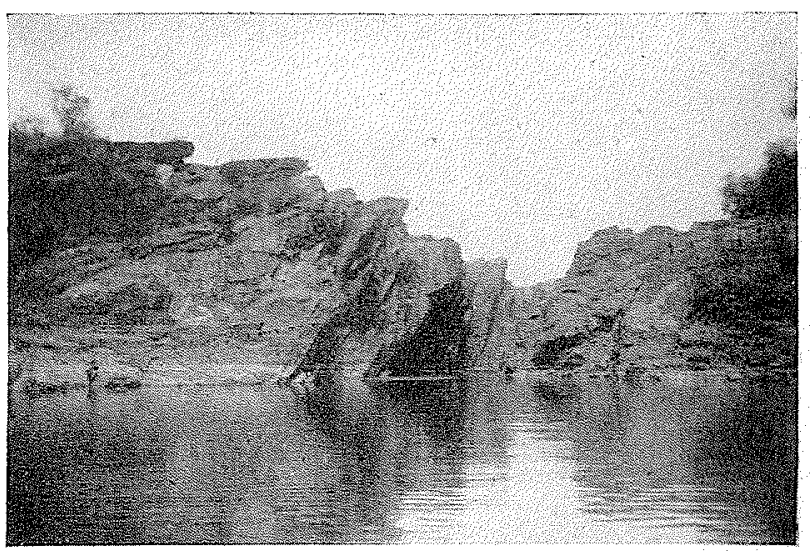

Fig. 9. - CHUTE DE TA RIVE DROTTE DF ra SANAGA A I'ÉTHAE, VUE D'AVAL 


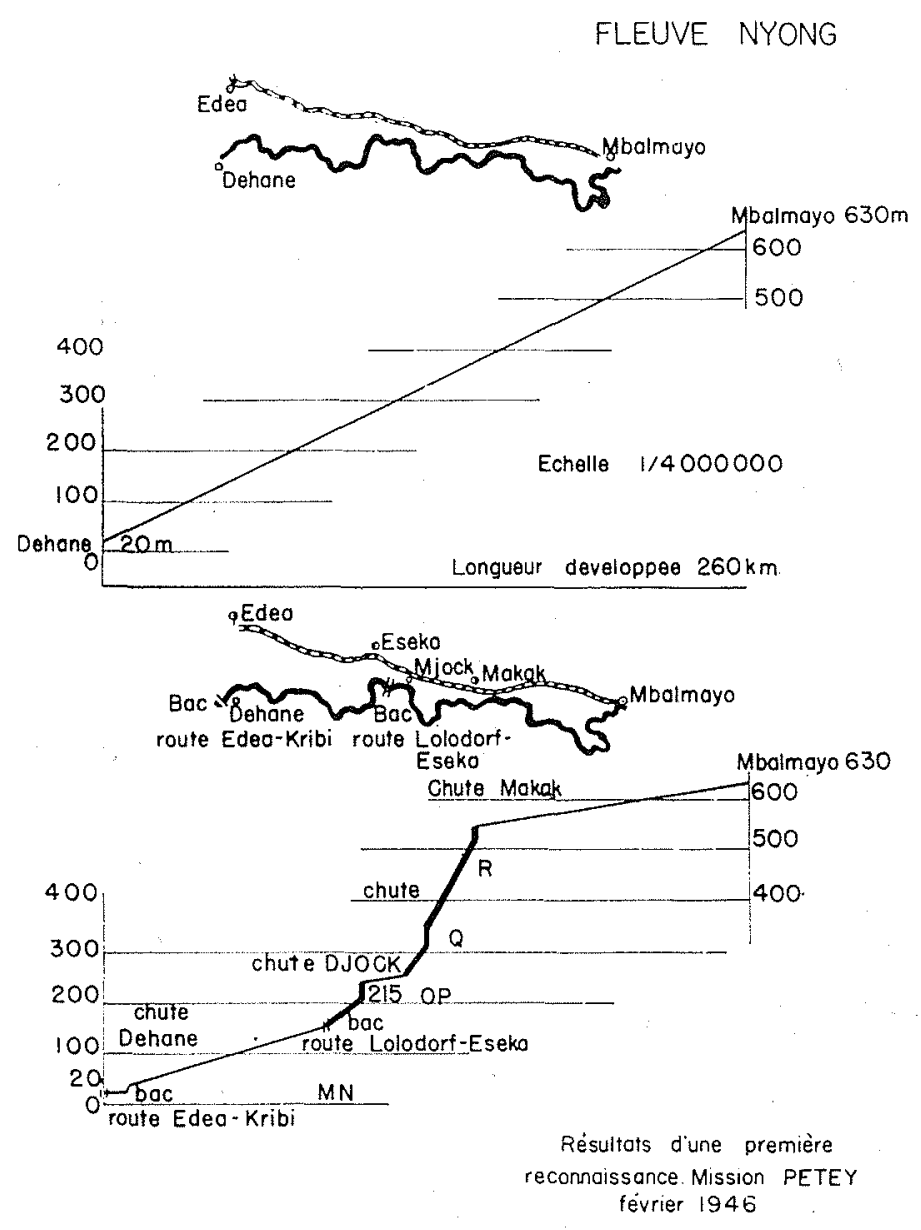

fig. 12. - REsurats D'UNE PREMERE RECONNAISSANCE, T.FS SECTIONS MN, $O P$ ET $Q R$ PRESTNTENT UYE PENTE GENÁ-

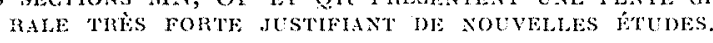

(fig. 11) que, dans la plus grande partie de son cours, entre M'balmayo et Abong-M'bang, ce fleuve présente une très faible pente, c'est d'ailleurs le principal bief navigable du Cameroun. Dans cette partie ses affluents peu importants ont également une très faible pente.

Après M'balmayo il n'en est plus de même et au sud de Makak le fleuve qui est encore à l'altitude de plus de $600 \mathrm{~m}$. descend dans des gorges profondes vers la mer. La différence de nivean atteint près de $600 \mathrm{~m}$. sur une longueur développée de $160 \mathrm{~km}$. Là encore, nous nous trouvons devant un fleuve extrèmement intéressant du point de vue de l'utilisation possible de l'énergie. hydroélectrique. Les échelles que nous avons fait poser au pont du Nyong à M'balmayo et au bac de la route de Lolodorf sont encore lues trop irrégulièrement depuis un an à peine pour que l'on puisse utiliser des renseignements précis. Il en est de même des premiers jaugeages exécutés aux moyennes eaux. Si cependant on admet

(6) Mission PETry. le mème débit moyen an kilomètre carré que pour la Sanaga, la puissance théorique brute disponible dans les chutes du Nyong serait comprise entre $450.000 \mathrm{cv}$. et $5.400 .000 \mathrm{cv}$.

Pour donner une idée des difficultés d'accès et d'études dans des régions inconmues nous avons indiqué (Pl. 12) l'état de nos connaissances en août 1945 : le profil en long constituait une ligne droite théorique.

La Mission que nous avons envoyée sur place en février 1946 (6) a pu accéder, en quelques points seulement, mais nous pouvons déjà avoil: une idée plus précise du profil en long du fleuve (pl. 12).

On voit que des zones MN, OP, QR semblent présenter une très forte pente, des reconnaissances ultérieures seront donc nécessaires.

La planche 13 reproduit la disposition des deux chutes verticales de Makak et de N'Jock, il $y$ en a d'autres certainement mais pour $y_{\text {}}$ accéder, il sérait nécessaire de frayer des chemins dans cette partie de la grande forêt. Lì encore, sous réserve d'études complémentaires, quelques grands barrages permettraient l'utilisation d'une notable partie de cette énergie.

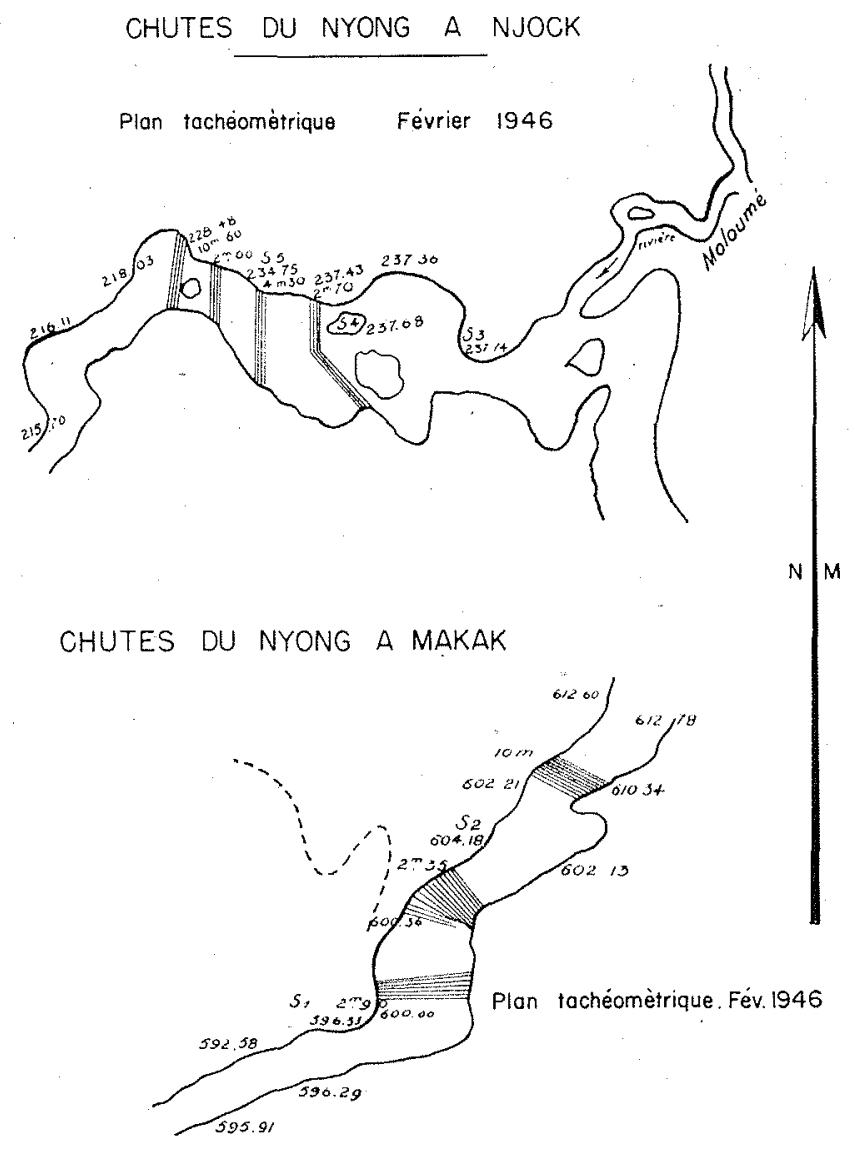

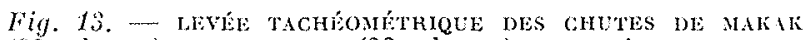

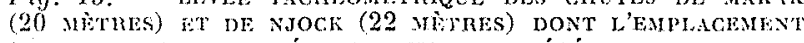
EST IXURUÉ A LA FYGRE PRÉCÉDENTE. 


\section{ESSAI DE PROFIL EN LONG DU N'TEM}

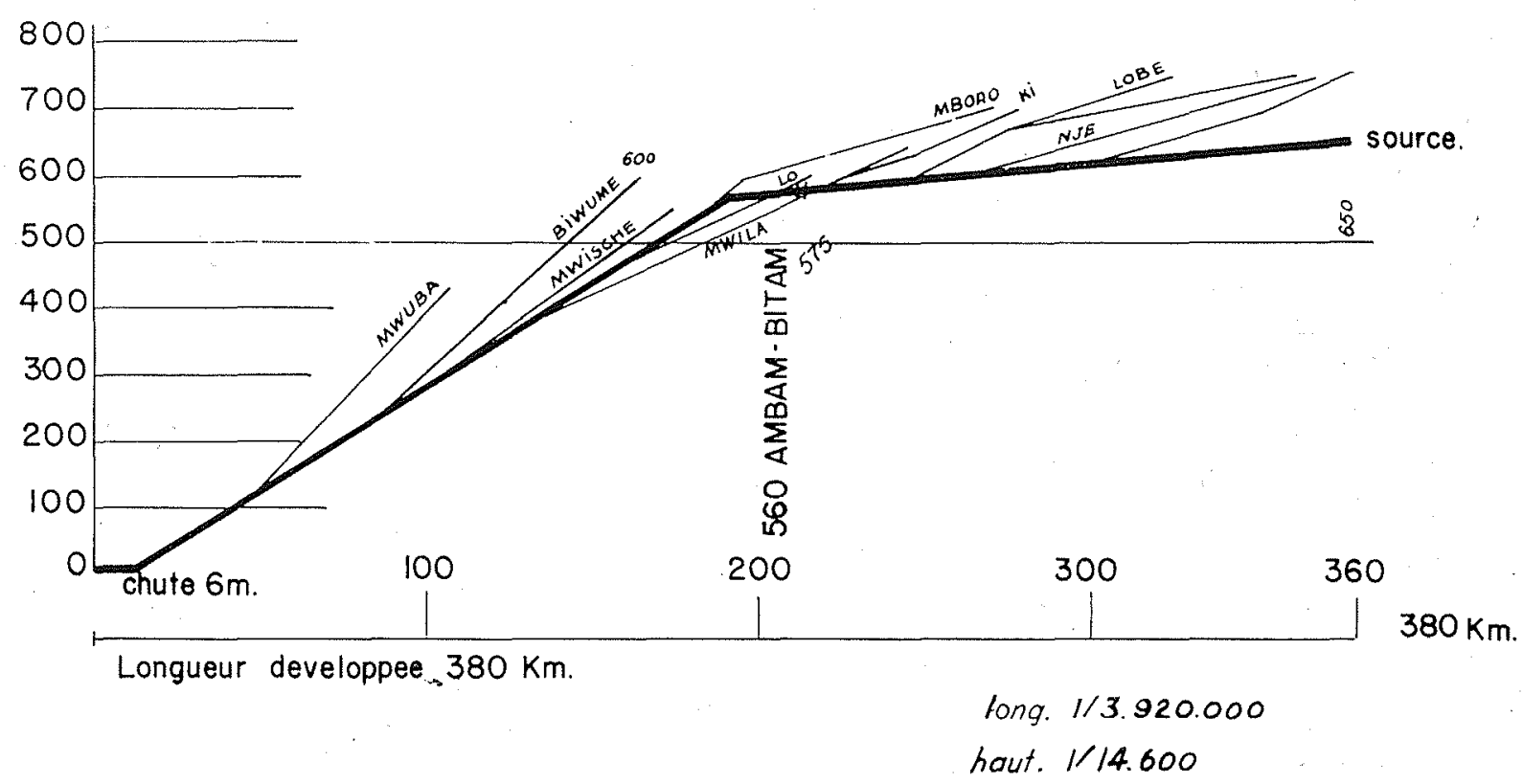

Juillet 1946

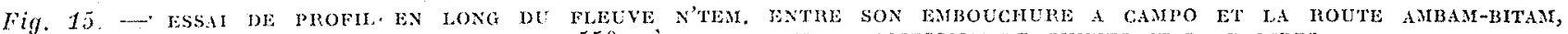

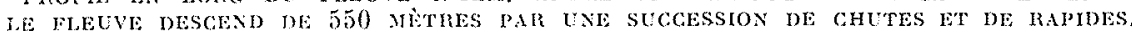

\section{CHAPI'TRE III}

\section{Bassin du N'tem}

Le N'tem est un fleuve qui se trouve, comme nous l'avons dit, à la limite de la frontière du Gabon. Le bassin s'étend sur $30.000 \mathrm{~km}^{2}$ dont une part importante se trouve en Afrique Equatoriale Francaise (fig. 14).

Nous avons établi un essai de profil en long qui présente des caractéristiques analogues au Nyong: une partie supérieure où le N'tem a une très faible pente ainsi que ses affluents puis, à partir de la route Ambam-Bitam le fleuve descend rapidement par des chutes et des rapides pour se jeter dans l'Océan après un parcours de $200 \mathrm{~km}$ où il présente une dénivellée de plus de $550 \mathrm{~m}$. Cette région est située en pleine forêt tropicale malheureusement très peu connue (fig. 15). L'administrateur Granier a cependant signale une chute verticale d'une quarantaine de metres de hauteur qui se trouverait au Surl de Nyabessam. Nous nous proposons. de faire reconnaître celte région afin de permettre de localiser les zones de tres forte pente; par la suite on pourra avoir une idée exacte des possibilités d'aménagement hydroélectrique de ce fleuve, dont la puissance théorique brute doit varie' entre $410.000 \mathrm{cr}$ et $4.950 .000 \mathrm{cv}$.

\section{POSSIBILITES DE PRODUCTION D'ENERGIE HYDROELECTRIQUE}

Avant de passer en revue les autres petits bassins secondaires du Cameroun, nous croyons devoir appeler l'attention sur cette zone de superficie relativement limitée qui comprend les

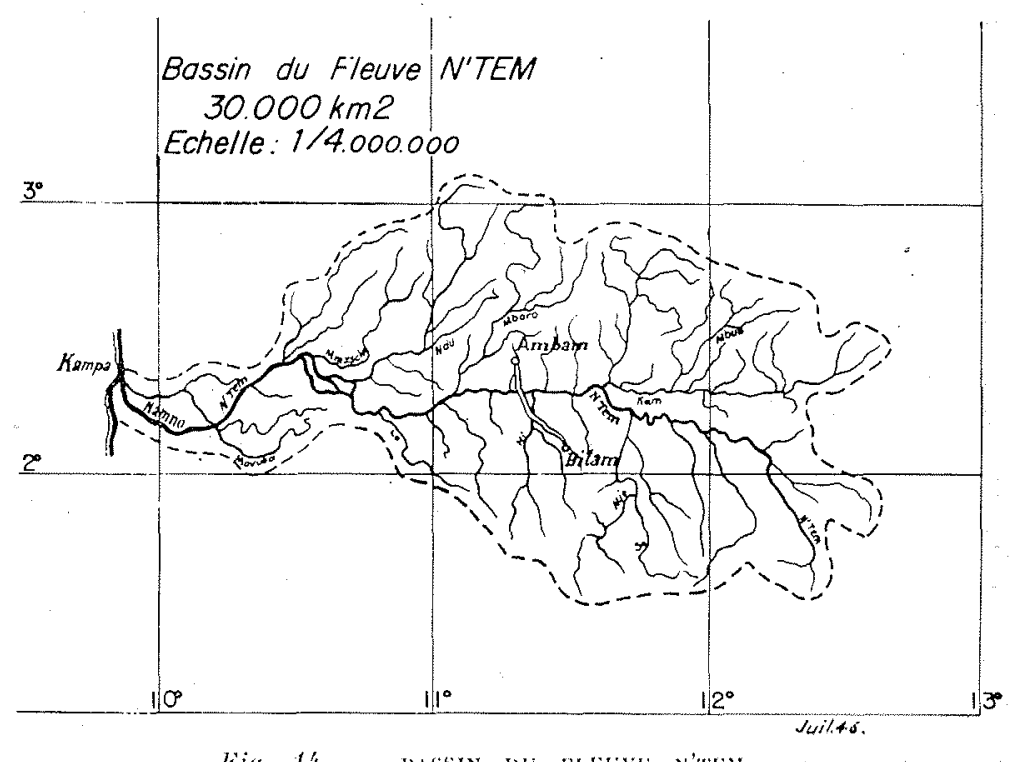

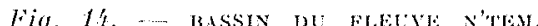

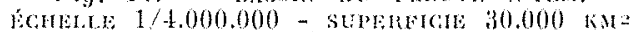


Lrois partiee à forte pente de la Sanaga, du Nyong et du N'tem représentant une puissanca hydraulique considérable puisque, négligeant les affluents qui sont d'ailleurs très peu importants dans cette zone, on peut estimer que la puissance théorique brute de ces trois fleuves est comprise entre 2 et 25 millions de cv. (fig. 16).

Ces sections correspondent d'ailleurs à la limite du plateau cristallin central qui ne se trouve séparé de la còte que par une bande sédimentaire de largeur inégale, mais inférieure a 90 kilomètres.

$\mathrm{Si}$ nous rapprochons ces renseignements des idées que nous avions émises dans notre thèse (7) au sujet de l'utilisation possible des forces hydrauliques considérables du Moyen-Congo, du Gabon, et du Sud-Cameroun, on voit que les renseignements que nous avons recueillis par la suite confirment ces prévisions : la France dispose dans ces deux territoires de réserves d'énergie hydroélectrique d'aménagement très économique sur lesquelles elle pourra compter dès que la technique du transport d'énergie à très haute tension el a tres grande distance aura suffisamment fait de progrès. Les renseignements les plus récents confirment d'ailleurs que l'on peut espérer, d'ici quelques années, voir' réaliser des transports d'énergie à plusieurs. milliers de kilomètres. Il serait alors possible de disposer d'un appoint très important d'énergio a bon marché pour la France, certains autres pays de l'Ouest de l'Europe et l'Afrique du Nord.

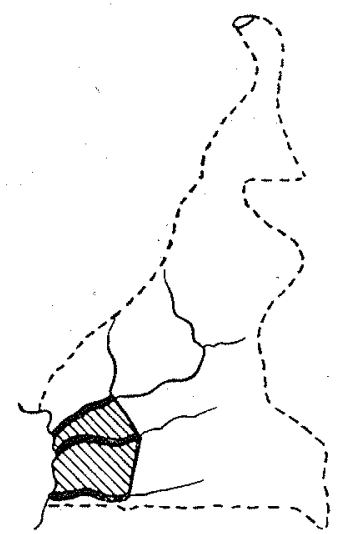

Fig. 16. - schéma FxaNa LES LMITES DE LA ZONE IA pLUS INTÉressaNTE AU PONT DE YUE RÉSERVE DE PUISSANGe HYDDOÉLCGTR-

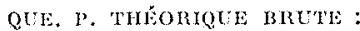
2 A 25 NILLIONS DE CV.

Un autre facteur peut encore contribuer a l'utilisation de ces réserves. Il y a lieu de signaler en effet que le débit de ces cours d'ean cor'respond à des régimes de pluies très différents de ceux de l'Europe ou de l'Afrique du Nord (maximum pendant les mois de septembre, octobre, et intensité des précipitations 2 à $4 \mathrm{~m}$.). Cela peut constituer une compensation naturelle assurant une plus grande régularité dans la production de courant d'origine hydraulique.

(7) P. Darnaule these d'Inénietr-Docteur - Mai 1945. Faculte des Sciences de Paris (Lstrose, éditeur).

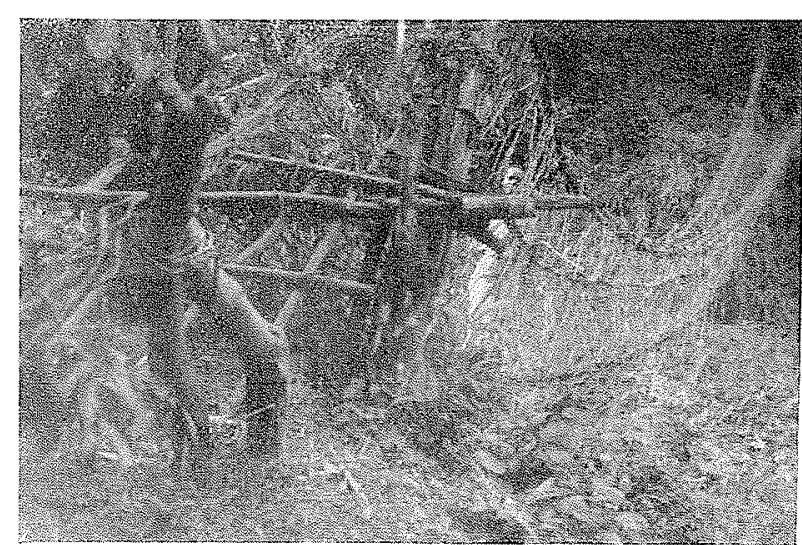

Fig. 21, UN PONT DE LAXES SUI LE N'KAM

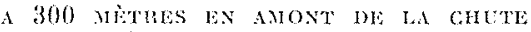

\section{CHAPITRE IV}

\section{Bassin du Wouri}

Le fleuve Wouri qui dessert la région de Douala présente un très vaste estuaire avant de se jeter dans l'Océan Atlantique. Son bassin versant est de $21.600 \mathrm{~km} \%$. Dans la partie nord, il recoit les émissaires du massif du mont Cameroun (4.070 m.) et du mont Manengoura (2.250), fig. 17 .

Ainsi qu'il ressort de l'examen du profil en long, fig. 18 , le Wouri et ses affluents qui prennent naissance dans la chaîne du Cameroun présentent dans la partie supérieure de leur cours une très forte pente ainsi que des chutes dont la plus importante est la chute d'Ekom sur le N'kam, affluent du Wouri. Sur 9 kilomètres, le N'kam descend de la cote 715 à la cote 325 avec une chute verticale de 70 à 80 mètres. Les pholographies (fig. 19, 20, 21) ainsi que le croquis fig. 22 montrent les dispositions d'ensemble de cette chute qui, dès que les besoins en énergie de la région la justifieront sera certainement utilisée, car elle se prête à un aménagement extrèmement économique. Les débits observés sont de l'ordre de $6 \mathrm{~m}^{3}$ à l'étiage, ce qui correspond à une puissance partielle de la chute de $4.800 \mathrm{cv}$ à l'étiage el 48.000 aux hautes eaux. Pour l'ensemble de la section utilisable d'environ 300 mètres de hauteur, avec dérivation de 9 kilomètres, 18.000 à $180.000 \mathrm{cv}$.

\section{CHAPITRE $V$}

\section{Bassin de la Benoue}

La Benote, afluent principal rive gauche du fleuve Niger qui prend sa source dans la partic nord du Territoire du Cameroun n'y a qu'une fraction peu importante de son bassin : $89.560 \mathrm{~km}^{3}$ (fig. 23 ). 


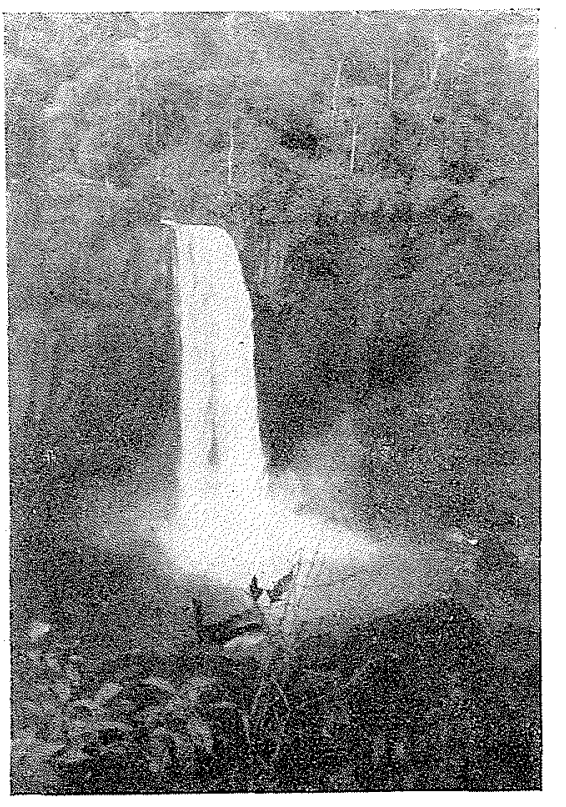

Ci-dessus : Fig $19,-$ IA CHUTY D'EKOY SUR LE N'KAM, PARTIE VERTRCALE DE 80 métries, VUE n'ENSEMBr.:

Ci-dessous : Fig. 17. - bassin versant Dt FLEUVE WOUBL ECHELLE $1 / 2.000 .000$. SUPERFICIE $21.600 \quad \mathrm{KM}^{2}$

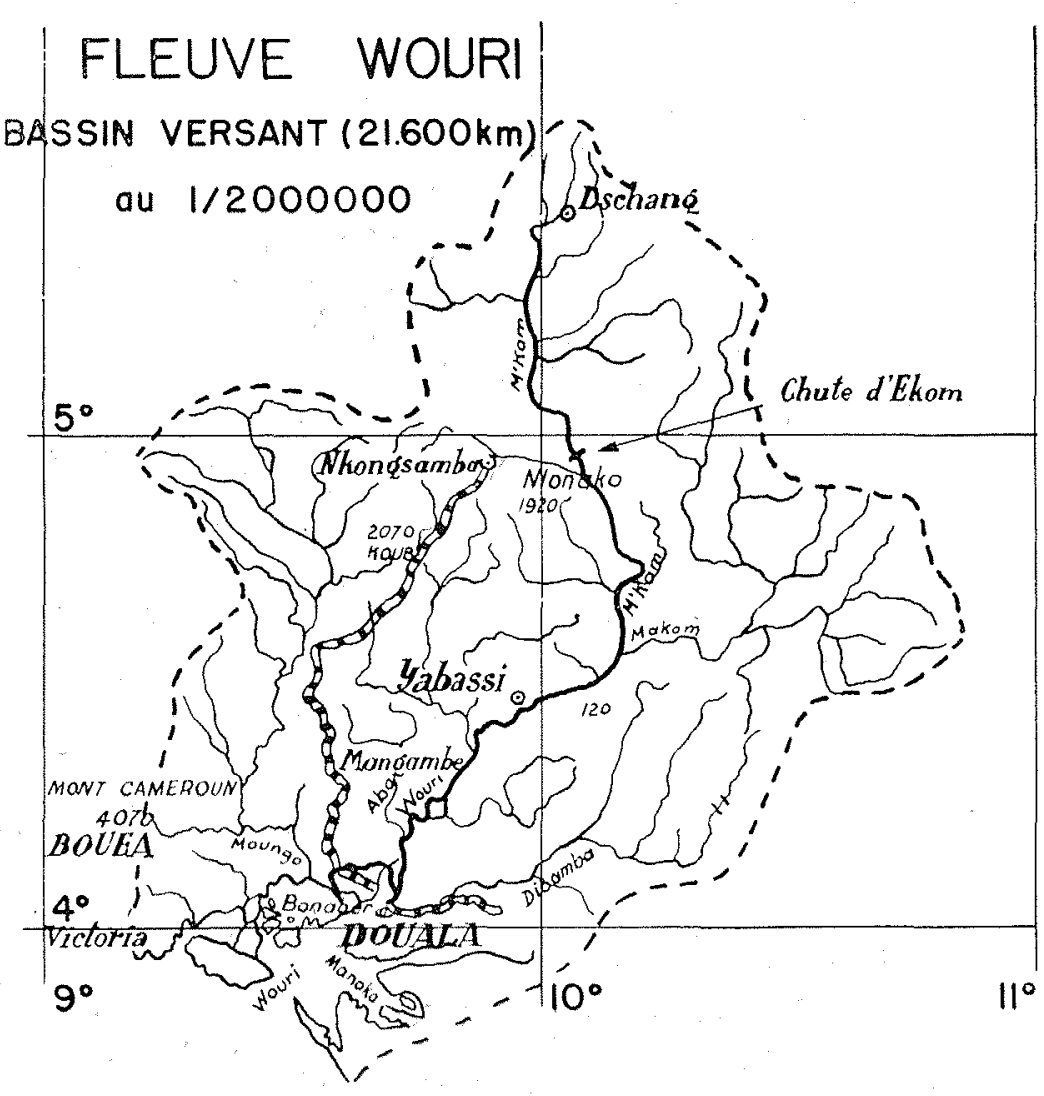

WOURI

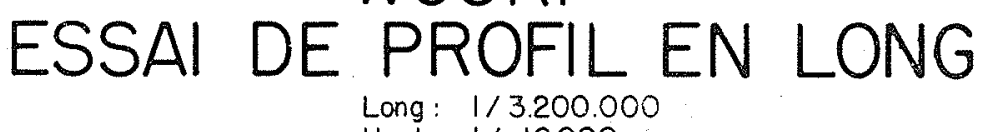

Haut : $1 / 16.000$

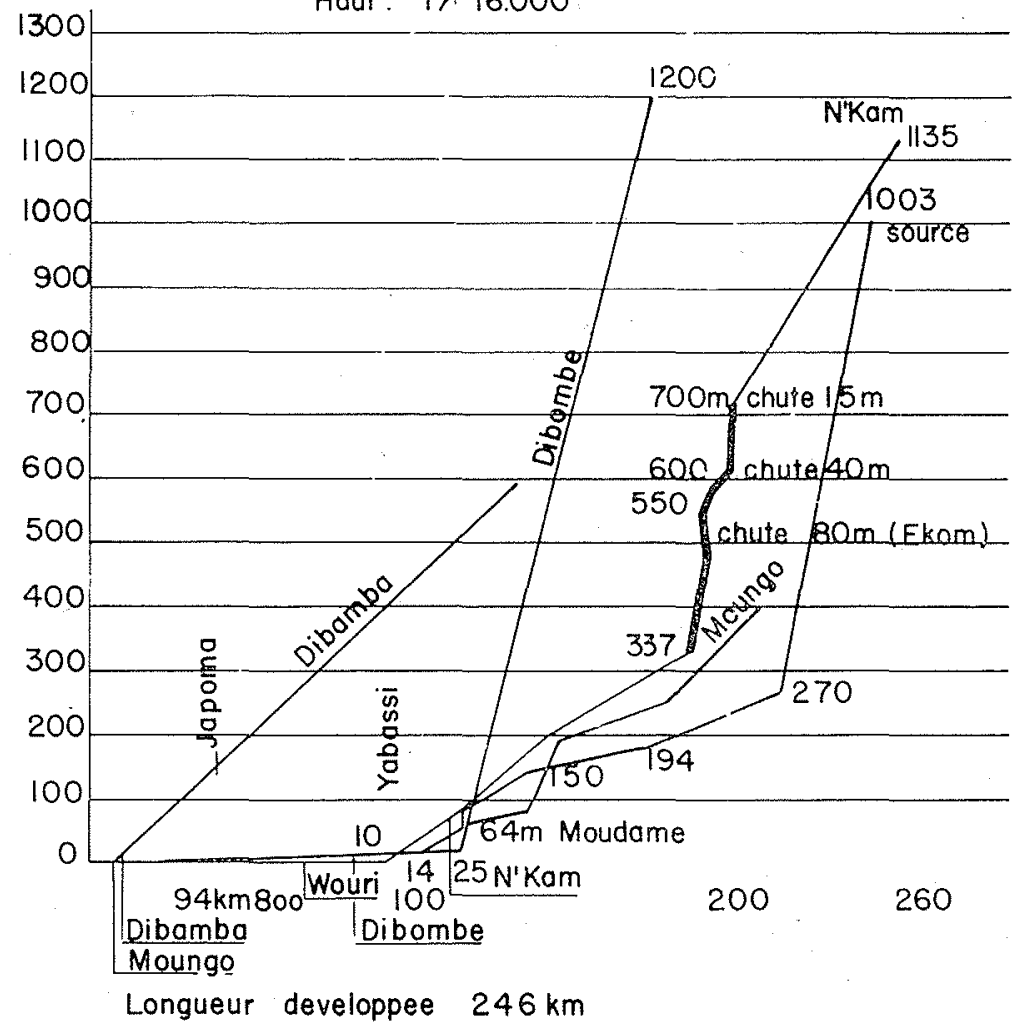

Ci-dessus : Fig. 18, - ESSAI de profth EN LONG DU FLEUV WOUR ET DE SBS

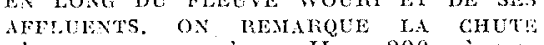
I'TKom ster re N'KaM. $H=290$ mÈtres SUR 9 FILOMLTRES

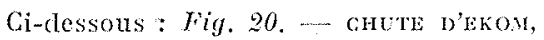
VUE PARTIELLE

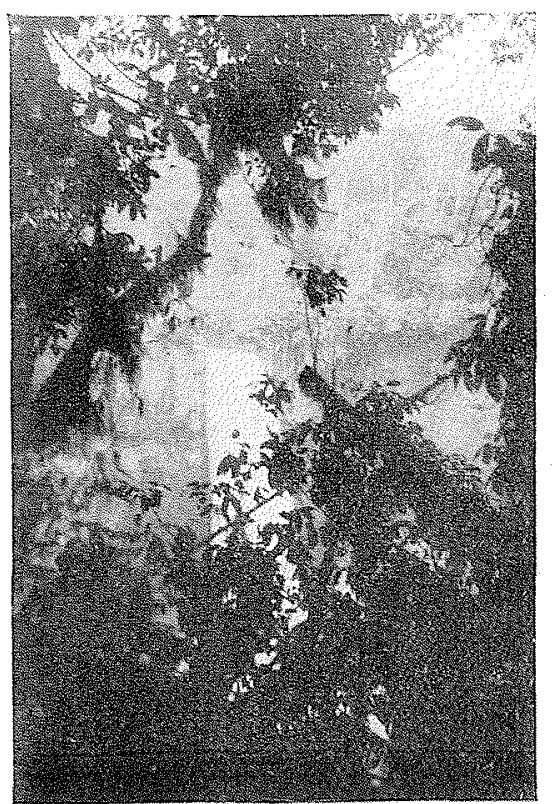


On voit d'après l'essai de profil en long (fig. 24) que la Benoue prenant sa source vers la coto 1.250 descend très rapidement à la cote 400 dans une région à peu près inconnue où il est vraisemblable que des chutes intéressantes pourront être découvertes. Il en est de même de son principal aflluent de la rive gauche, le Faro, qui prend sa source à 1.000 mètres et qui descend brusquement de la cote 900 à la cote 470 , enfin le Mayo Kebbi présente un accident aux chutes Gauthiot dont la hauteur est de 46 mètres; le reste de son cours se déroule suivant une très faible pente.

C'est par le Mayo Kebbi que les eaux du Logone risquent d'être un jour captées au profit de la Benoue, ce qui aurait pour effet d'assécher le lac T'chad.

A ce sujet, M..P. Havgov, Ingénieur' en chef des Mines vient de publier une étude très complète d'ou il ressort que ces craintes ne sont pas vaines.

Dans cette région quelques rares jaugeages ont pu ètre exécutés et des échelles limnimétriques mises en place. Les premiers résultats sont trop incomplets pour qu'il puisse en ètrs fait état dans cette première étude. Il sera cependant très utile de poursuivre ces travaux car la voie d'accès la plus économique dans cette région est la voie d'eau de la Benoue où des navires d'un tirant d'eau assez important peurent atteindre le port de Garoua pendant deux à trois mois de l'année. Faute de renseignements sur la propagation des crues de la partie supérieure de la Benoue, on se trouve à la merci d'une baisse rapide des eaux. En 1945 , la navigation a, en effet, été arrêtée par suite d'une baisse rapide mais de courte durée de la Benoue; ap'rès quelques jours de baisse, le débit a augmenté sensiblement, ce qui aurait permis d'assurer la navigation pendant un mois de plus.

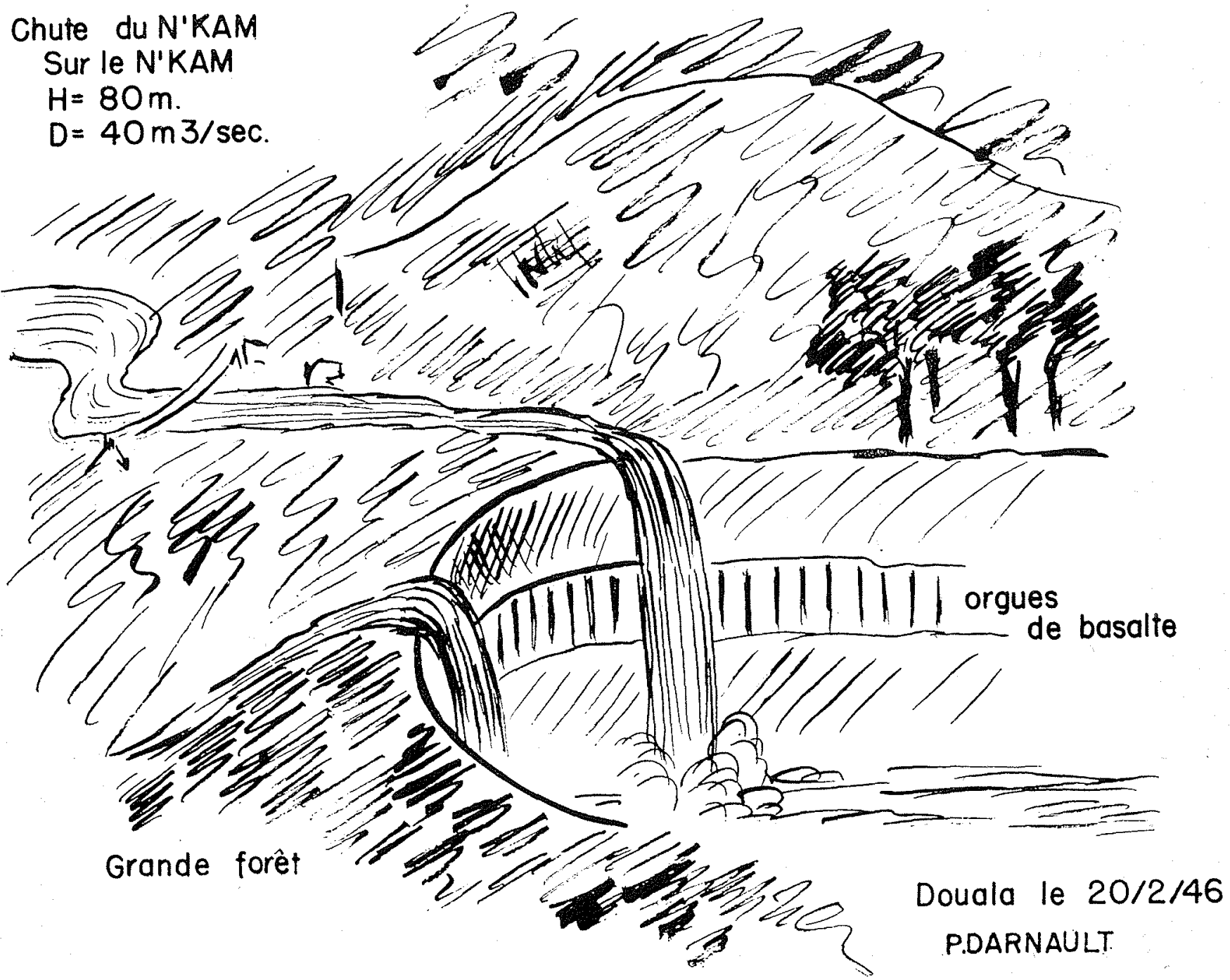

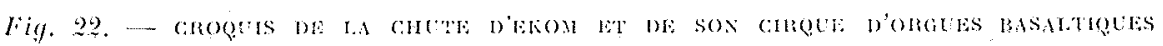




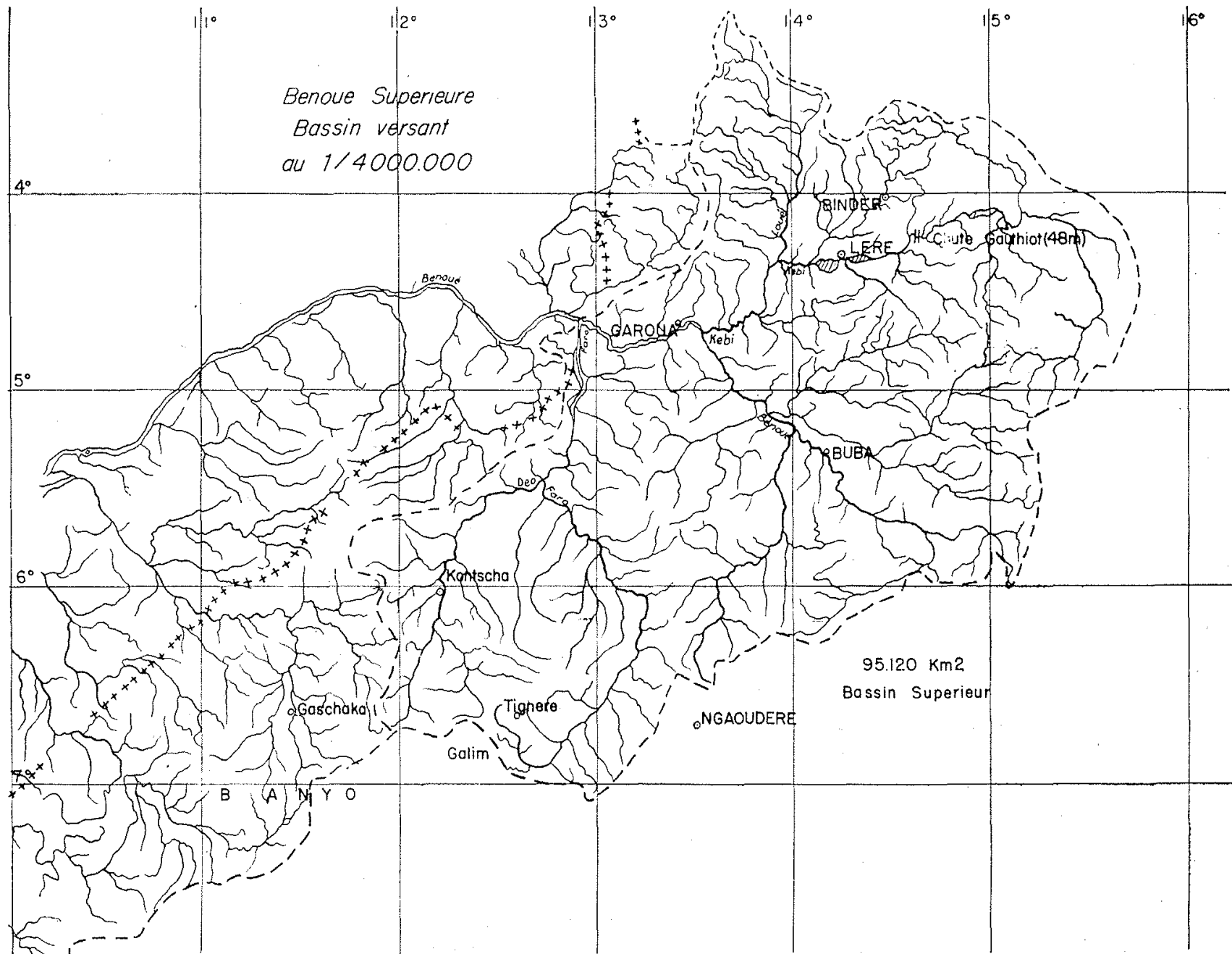

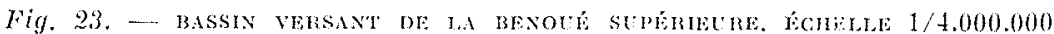

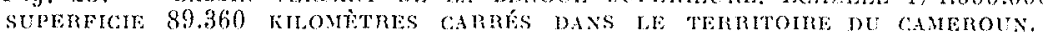

\section{CHAPITRE VI}

\section{Fleuves côtiers secondaires}

Nous citerons enfin quelques petits fleures cottiers dont l'importance du point de vue géographique physique est faible, mais qui peuveni présenter une certaine importance économique. Ce sont :

- La Lokoundji située entre le Nyong et le N'tem qui présente des chutes dans la partio supérieure de son cours.

- La N'kenke qui traverse la ville de Kribi avec une chute de 4 mètres à son embouchure.

- enfin la Lobe, cours d'eau plus important qui se jette dans l'Océan Atlantique par une chute de 13 mètres de hauteur au-dessus de laquelle s'étend une zone navigable d'une vingtaine de kilomètres. Il est possible que ce petit fleuve, dont l'embouchure pourrait servir de port pour des remorqueurs, devienne un centre d'embarquement pour les exploitations foreslières dont l'installation est prévue dans cetle région.

Le bassin versant de cette rivière est de $2.000 \mathrm{~km}^{2}$, ce qui correspondrait à une puissance utilisable pour la chute de l'ordre de $750 \mathrm{cv}$ à l'étiage et $7.500 \mathrm{cr}$ aux hautes eaux (fig. 25 et photographie fig. 26).

\section{CHAPITRE VII}

\section{Tributaires du Logone et du Congo}

\section{AFFLUENTS DU LOGONE}

Toute l'extrémité Nord du Cameroun-Tchad est sillonnée par une série d'affluents du Logone dont l'étude n'a pas encore été entreprise, car les premières échelles d'étiage nosées ont été 


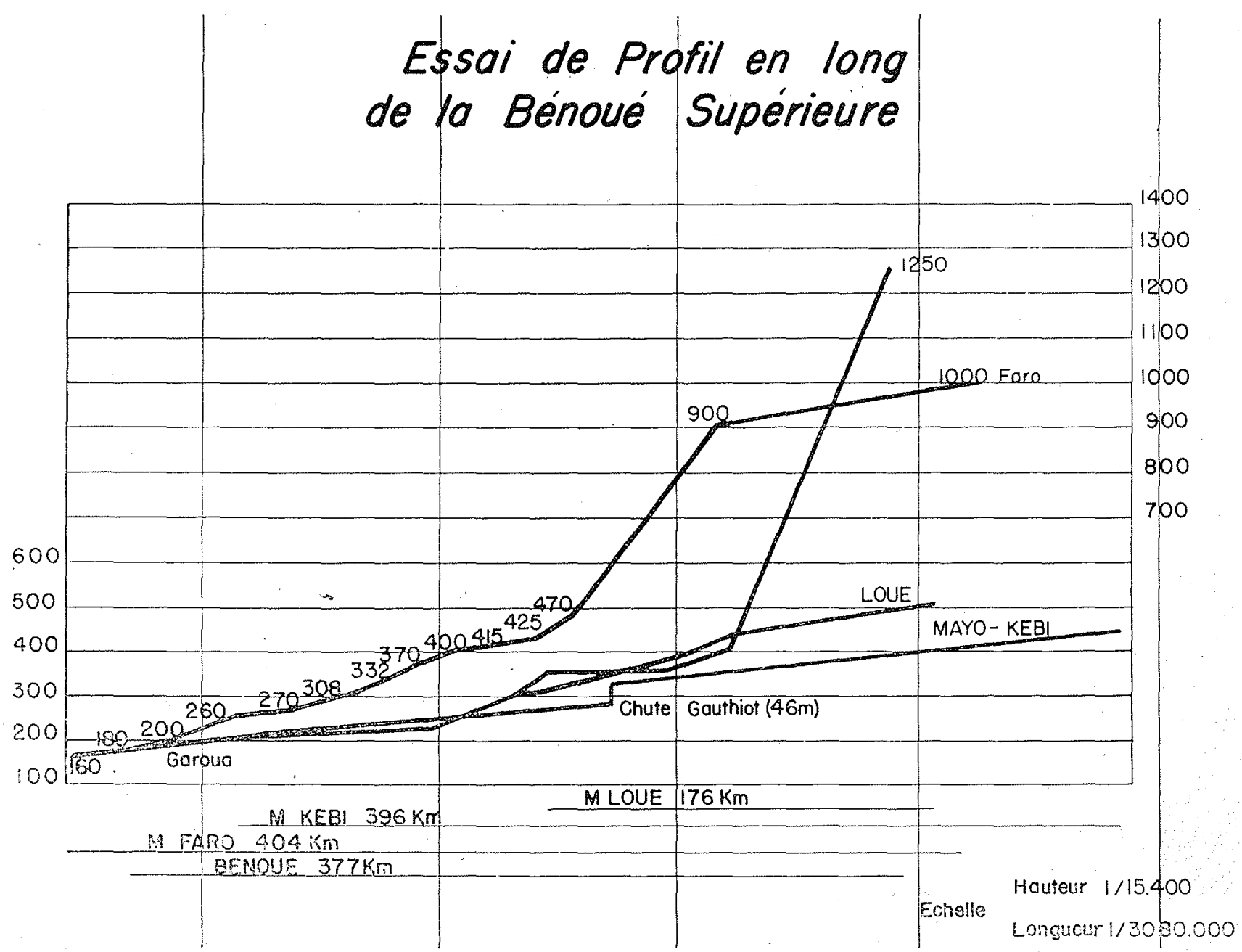

Fig. 24. - ESSAI DE PROFIL EN LONG DE LA RENOCÉ DT DE BES AFFLUENTS. mEMargVER LA CHUTE GAUTHIOT (46 M, ) SUR LE MAYO KEBBI.

emportées par les crues. Là peut-être plus qu'ailleurs, les cou'rs d'eau présentent des crues extrêmement rapides ; par contre, pendant une période sèche très lonģue les lits des cours d'eau sont complètement secs et les études qui seront faites dans celte région devront être liées à celles concernant l'hydrologie souterraine.

\section{AFFLUENTS DU CONGO}

Enfin à l'Est et au Sud, des sous-affluents de la Sanaga, affluent elle-même du fleuve Congo prennent leur source dans le territoire du Cameroun (Kadei, Dja). Des échelles d'étiage devront y être ultéieurement posées, mais l'étude de ces cours d'eau présente moins d'intérêt.

Tel est, rapidement esquissé, le tableau bien modeste encore de nos connaissances actuelles sur l'hydrologie du Cameroun. Il est certain que ces comnaissances qui sont à la base de tout travail important de Génie Civil, aménagement de force hydraulique, navigation, travaux dirt gation ou de distribution d'eau, devront etro complétées dans le plus court délai si lon veul éviter de paralyser le développement économique du Pays.

\section{III. -- PROGRAMMES DETUDES}

Nous avons cherché a constituer un réseau d'échelles limnimétriques et à établir des stations de jaugeages en un cerlain nombre de points des différents cours d'eau limitant provisoirement les mesures au voisinage des centres habités, afin d'assurer un meilleur contrôle des lectures. Ces études encore bien fragmentaires et d'une précision très relative devront être poursuivies régulièrement au cours des prochaines années et pourront, sans doute être assurées par les Services techniques locaux.

Toutefois, il est à craindre que l'intérêt de telles mesures n'échappe à des techniciens déjà 
accablés de travaux très divers. On ne peut raisonnablement demander à ces derniers de s'attacher à des recherches scientifiques en $y$ consacrant le temps nécessaire et nous avons déja constaté ce fait il $y$ a bien longtemps en Afrique Equatoriale Francaise.

Seul un Organisme de Recherche Scientifique nous semble qualifié pour organiser, animer, contròler ces études dans toutes les colonies. II devait centraliser les résultats, les comparer. avec les études des pays étrangers voisins et pourrait tirer par la connaissance des relevés météorologiques et de la géologie des conclusions scientifiques intéressantes : coefficients d'écoulement, débouchés d'ouvrages d'art, propagation des crues, débit d'étiage et de crues, hydrologie souterraine, irrigation, caractéristiques physiques et chimiques des eaux.

\section{CONCLUSIONS}

Le Cameroun qui présente toutes les gamme: depuis le lac Thad jusqu'à la frontière du Gabon : régime très varié des pluies, variété des altitudes, de la nature des terrains, de la végétation zones de savane seche, de savane boisée, de grande forèt) parait appelé à un développon ment économique rapide. Toutefois, cette mise en valeur dépendra dans une certaine mesure des études hydrologiques qui, complètement délaissées jusqu'ici vont ainsi présenter un intérèt pariculier. Dans ces conditions nous ne satirions trop insister pour que l'organisation esquissée soit définitivement créce.

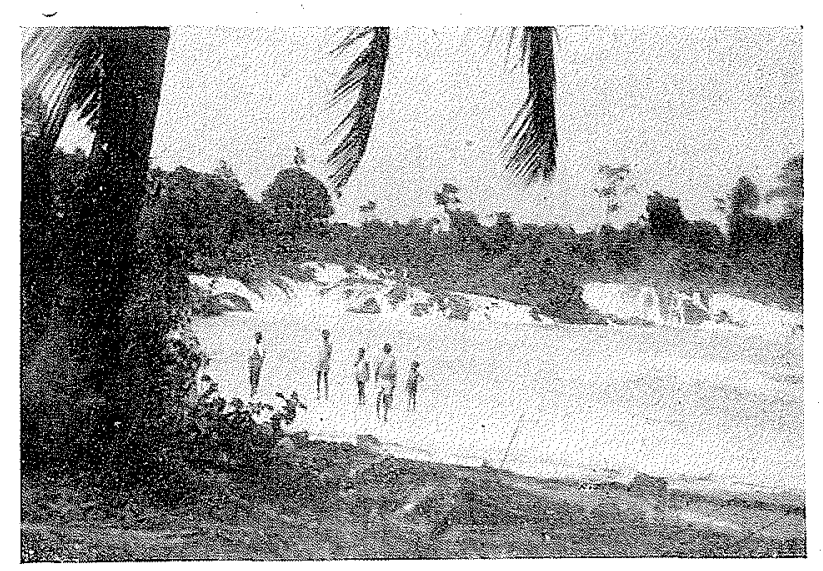

Fig. 26. - chute de Fieve cotren robs

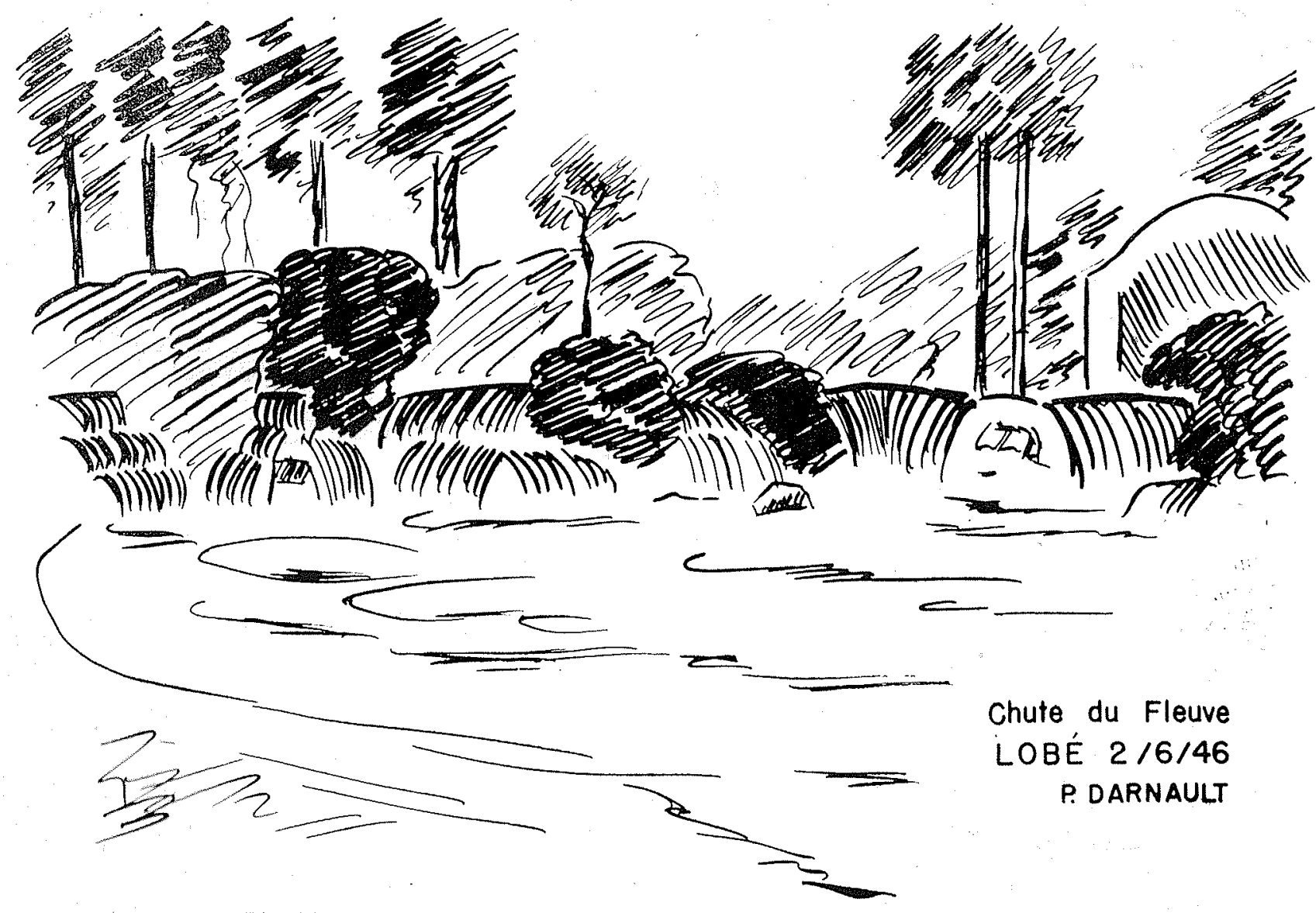

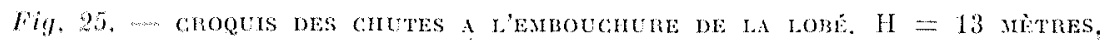

